\title{
Plasticity in the Frequency Representation of Primary Auditory Cortex following Discrimination Training in Adult Owl Monkeys
}

\author{
G. H. Recanzone, ${ }^{1}$ C. E. Schreiner, and M. M. Merzenich \\ Coleman Laboratory, Departments of Otolaryngology and Physiology and Keck Center for Integrative Neuroscience, \\ University of California at San Francisco, San Francisco, California 94143-0732
}

\begin{abstract}
Previous studies have shown that the tonotopic organization of primary auditory cortex is altered subsequent to restricted cochlear lesions (Robertson and Irvine, 1989) and that the topographic reorganization of the primary somatosensory cortex is correlated with changes in the perceptual acuity of the animal (Recanzone et al., 1992a-d). Here we report an increase in the cortical area of representation of a restricted frequency range in primary auditory cortex of adult owl monkeys that is correlated with the animal's performance at a frequency discrimination task.

Monkeys trained for several weeks to discriminate small differences in the frequency of sequentially presented tonal stimuli revealed a progressive improvement in performance with training. At the end of the training period, the tonotopic organization of Al was defined by recording multiple-unit responses at 70-258 cortical locations. These responses were compared to those derived from three normal monkeys and from two monkeys that received the same auditory stimuli but that were engaged in a tactile discrimination task. The cortical representation, the sharpness of tuning, and the latency of the response were greater for the behaviorally relevant frequencies of trained monkeys when compared to the same frequencies of control monkeys. The cortical area of representation was the only studied parameter that was correlated with behavioral performance. These results demonstrate that attended natural stimulation can modify the tonotopic organization of $\mathrm{Al}$ in the adult primate, and that this alteration is correlated with changes in perceptual acuity.
\end{abstract}

[Key words: auditory cortex, primate, plasticity, behavior, physiology, discrimination training]

Studies within the somatosensory system have shown that partial peripheral dennervation and restricted natural stimulation can result in a reorganization of the topographic representation of the body surface in the cerebral cortex (see Merzenich et al., 1990; Recanzone and Merzenich, 1992). Similarly, reorganization of the topographic representation of the cochlea in the primary auditory cortex has been demonstrated following par-

Received Nov. 27, 1991; revised June 23, 1992; accepted July 9, 1992.

We thank G. T. Hradek, M. L. Sutter, and R. E. Beitel for their important contributions to this work, W. M. Jenkins for insightful discussions regarding the behavioral procedures, and Bret E. Peterson for the computer software used in the data analysis. Funding was provided by NIH Grants NS-10414, GM-0774 and ONR N00014-91-J-1317, Hearing Research Inc., and the Coleman Fund.

Reprint requests should be addressed to M. M. Merzenich at the above address. All other correspondence should be addressed to G. H. Recanzone, Laboratory of Sensorimotor Research, Building 10, Room 10C101, National Institutes of Health, Bethesda, MD 20892.

Copyright@1993 Society for Neuroscience $0270-6474 / 93 / 130087-17 \$ 05.00 / 0$ tial cochlear lesions (Robertson and Irvine, 1989; Irvine et al., 1991). Tuning properties of single cortical neurons have been shown to be altered by conditioning paradigms (Olds et al., 1972; Disterhoft and Stuart, 1976; Kitzes et al., 1978; Ryugo and Weinberger, 1978) and pharmacological manipulations (Ashe et al., 1989; McKenna et al., 1989; Metherate and Weinberger, 1990; see Weinberger et al., 1990, for review). Although the cortical tonotopic map was not explicitly studied, the functional plasticity at the single neuron level suggests that the tonotopic representation of the cochlea in the cortex may be altered as a result of the behavioral training.

Reorganization of cortical representations has been argued to be continuous throughout life and to reflect an individual's ability to acquire new skills and behaviors (Merzenich et al., 1988, 1990). Improvements in performance have been demonstrated for both simple auditory detection and discrimination tasks (Zwislocki et al., 1958; Sinnott et al., 1985; Prosen et al., 1990), and similar improvements in performance on tactually based tasks have recently been demonstrated to parallel changes in the functional organization of somatosensory cortical areas $3 \mathrm{a}$ and 3b (Recanzone et al., 1992a-d). The demonstrations that auditory cortical neurons can alter their frequency-specific response properties by either peripheral dennervation or behavioral conditioning suggested to us that the types of cortical reorganization seen in the somatosensory cortex also occur in primary auditory cortex. Specifically, we hypothesized that the functional organization of $\mathrm{AI}$ is altered as a consequence of an animal's improvement in auditory discrimination ability. To test this hypothesis, three adult owl monkeys were trained at an auditory frequency discrimination task for several weeks. The tonotopic representation of the primary auditory cortex was defined electrophysiologically after significant improvements in performance were measured. The response properties of these neurons were then compared to those of normal monkeys, as well as to those of monkeys that had received the same auditory stimulation but were engaged in an unrelated tactile task. The electrophysiological data were then analyzed to determine if any simple measure of the cortical representation of the frequencies used in the behavioral paradigm was correlated with the improved behavioral performance.

\section{Materials and Methods}

Animals. Ten adult owl monkeys of both sexes were used. Animals were judged to have normal hearing based on evoked potential recordings (ABR). The outer ear canal was determined to be free of obstruction and infection. Monkeys were given free access to water and were food deprived for a 21-22 $\mathrm{hr}$ period prior to each psychophysical testing session. Supplemental fruit was given at the end of each testing session to maintain body weight between $85-95 \%$ ad lib. The care and treatment 
of these monkeys were in accordance with the NIH Guide for the Care and Use of Laboratory Animals (revised 1987).

Psychophysics. The psychophysical apparatus and methods have been described in detail (Recanzone et al., 1991). Five monkeys were trained to detect a difference in the frequency of sequentially presented pairs of tone pips. Monkeys were trained in an acoustically transparent test cage housed within a single-walled acoustic chamber lined with echo-attenuating foam. Auditory stimuli were delivered in the free-field from a single speaker (Realistic model 14-1996) located directly over the animal's head. All aspects of the stimulus generation and data collection were automated and under the control of a microcomputer.

Monkeys were trained to initiate a trial by contacting a hand mold (monkeys OM1, OM2, OM3, CM1) or bar located outside of the test cage (OM4 and OM5), or by pulling a lever located inside the test cage (CM2). In each case, this observing response located the animal's head in a stereotyped location and orientation. Head position was monitored continuously with a video camera. Tone pip pairs were presented every $650 \mathrm{msec}$. Each tone pip had a duration of $150 \mathrm{msec}(3 \mathrm{msec}$ rise/fall), with the onset of the second pip occurring $50 \mathrm{msec}$ after the offset of the first. The frequency of the first tone pip was constant for a given frequency discrimination task. Four different frequency ranges were tested: $2.5 \mathrm{kHz}$ task ( $2511 \mathrm{~Hz}$ standard), $3 \mathrm{kHz}$ task $(3188 \mathrm{~Hz}$ standard), $5 \mathrm{kHz}$ task $(4765 \mathrm{~Hz}$ standard), and $8 \mathrm{kHz}$ task (7950 IIz standard). Each monkey was extensively trained on at least one of these four tasks, and most were also tested on at least one other task on a few sessions spaced throughout the training period. The comparison, or S1, stimulus consisted of both tone pips of the pair being equal in frequency (the standard). For the target, or S2, stimulus, the frequency of the second tone pip was different from the frequency of the standard. Monkeys were required to maintain contact with the mold, bar, or lever throughout the presentations of the S1 stimuli, and to release contact upon detection of the $S 2$ stimulus within a $450 \mathrm{msec}$ reward window beginning $100 \mathrm{msec}$ after $\mathrm{S} 2$ stimulus onset. Failures to release within this reward window were scored as a "miss" and were punished by a 1-5 sec timeout. Releases before this time were scored as a "false-positive" and also resulted in a time-out. Releases within the reward window were scored as a "hit" and the monkey received a $45 \mathrm{mg}$ banana-flavored food pellet (Bio Serve). Six to ten different S2 stimulus frequencies were presented on at least 20 trials during a given session. These frequencies were chosen such that the monkey received a reward on $65-75 \%$ of all trials. Each monkey would perform $400-750$ trials in a daily session.

The number of SI stimulus presentations (bins) on a given trial was pseudorandomly determined to be between 2 and 11 . The probability that the next stimulus would be an \$2 (approximately 0.3 ) did not vary during the course of a single trial. In the condition that $11 \mathrm{~S} 1$ stimuli were presented, the 12 th stimulus was always the $S 2$. These trials were not included in the data analysis, as they were predictably the $\mathrm{S} 2$ based on time alone. Trials in which the monkey made a response in bins 1 and 2 were similarly omitted as they were always the $\mathrm{S} 1$. The performance $(P)$ for each $\mathrm{S} 2$ frequency was defined by

$$
P=H * S \text {, }
$$

where $H$ is the hit rate for that frequency $(H=$ \# correct responses/\# presentations) and $S$ is the safe rate correction factor $(S=1-$ falsepositive rate). The psychometric function for each session was determined by plotting $P$ as a function of the frequency difference between the $\mathrm{S} 1$ and S2 stimuli (delta frequency: $\Delta F$ ). Threshold was defined from this function as the $\mathrm{S} 2$ frequency at which $P=0.50$. This is a reliable measure for discrimination performance that is essentially unaffected by false-positive rates below 15\% (see Recanzone et al., 1991, 1992a). The slope of the psychometric function was defined as the slope of the line connecting the data points immediately above and below threshold. Threshold was defined in all sessions in which at least one $\mathrm{S} 2$ stimulus had a performance value below 0.50 and the false-positive rate for the session was below $15 \%$. The psychophysical data for several different S2 frequencies were subjected to signal detection analysis as described by Green and Swets (1966) for five consecutive training sessions spaced throughout the total training period (see Results). This analysis measures the difference in the response between the $S 1$ and $S 2$ stimuli in units of standard deviation and is independent of changes in the subject's internal criteria.

Three classes of control trials vertified that responses were based on the perception of the frequency of the auditory stimulus. On approximately $2 \%$ of the trials, the S2 stimulus was not presented until after $11 \mathrm{~S} 1$ presentations (described above). On these trials, a release response after the 12th stimulus always resulted in a reward; however, the performance at any given frequency was comparable regardless of the stimulus presentation bin. On $0.5-1.0 \%$ of the trials, the $\mathrm{S} 2$ stimulus generated by the computer had a delta frequency of zero $(\mathrm{S} 1=\mathrm{S} 2)$. Monkeys under stimulus control never responded in this condition. Finally, monkeys did not respond on trials in which the speaker was disconnected yet all other aspects of the trials were identical.

In order to prevent intensity cues as a function of frequency consequent to changes in the location of standing waves present in the free field, the intensity of each tone pip was varied independently over a $10-12 \mathrm{~dB}$ range. The intensity of the stimuli centered in this range was measured at three or four different frequencies at 12 separate locations throughout the sound field for each of the four tasks (Table 1). These locations were chosen to sample the area occupied by the monkey's head during a session and formed a cube of approximately $5 \mathrm{~cm} / \mathrm{side}$, with four additional measurements within this cube. There was no statistically significant difference in the intensity measured throughout the sound field between any of the four tested frequencies for each task (two-tailed $t$ test; $p>0.10$ ). Furthermore, repeated measures throughout the course of this study showed no statistical differences over time. The spectral content of these signals was measured in the free field to determine the potential contributions of each harmonic of the signal. The highest-intensity harmonics were always $40-50 \mathrm{~dB}$ less than the intensity of the fundamental. Finally, the head position of each monkey was viewed continuously with a video camera on each session, and analysis of head position with the behavioral response was made from several videotaped sessions in each monkey. This analysis showed no consistent relationship between the location or small movements of the monkey's head with the presentation of stimuli or with the behavioral response.

In addition to the five monkeys trained at the auditory frequency discrimination task, one monkey (CM1) was trained to detect a change in the frequency of a sinusoidal tactile stimulus (monkey E-2 of Recanzone et al., 1992a-d). The same auditory stimuli as used in the 8 $\mathrm{kHz}$ task were presented simultaneous to the presentation of the tactile stimulus, but did not give any indication of reward availability. Monkey CM2 was trained to perform a tactile vibration detection task in the presence of the same auditory stimuli used in the $5 \mathrm{kHz}$ task. These monkeys represent passive-stimulation controls.

Electrophysiology. The primary auditory cortex was defined electrophysiologically in the seven monkeys trained in a psychophysical task and in three normal owl monkeys (N1, N2, and N3) that had not been trained at any task. Anesthesia was induced with a halothane $(3 \%)$ : nitrous oxide $(72 \%)$ : oxygen $(25 \%)$ gaseous mixture. The femoral vein was cannulated and sodium pentobarbital was given intravenously to maintain an areflexic state of anesthesia $(28 \mathrm{mg} / \mathrm{kg}$ induction, $1-5 \mathrm{mg} / \mathrm{hr}$ maintenance). Penicillin G $(30,000 \mathrm{U} / 24 \mathrm{hr})$ and atropine sulfate $(0.1 \mathrm{mg}$ $\mathrm{kg} / 12 \mathrm{hr}$ ) were given intramuscularly. Lactated Ringer's solution with $5 \%$ dextrose was continuously infused $(2-5 \mathrm{ml} / \mathrm{hr})$. Heart rate, respiration rate, and other vital signs were monitored. Core body temperature was maintained at $37^{\circ} \mathrm{C}$ with a thermostatically controlled heating blanket. A craniotomy exposed the relevant cortical region, the dura was removed, and a well of silicon oil was constructed to bathe the cerebral cortex continuously. A $40 \times$ image of the cortical surface was taken either photographically or with a digitized video image. All electrode penetrations were parallel to each other. The electrode insertion points were reproduced onto the image of the cortical surface with reference to the vasculature. Electrode penetrations away from the sulcus were made only to a single depth corresponding to the middle cortical layers (500-900 $\mu \mathrm{m}$ below the cortical surface).

The stimulus generation and data acquisition methods have been described in detail elsewhere (Schreiner and Mendelson, 1990). Briefly, single tone pips of $50 \mathrm{msec}$ duration ( $3 \mathrm{msec}$ on/off ramp) were generated by a microprocessor (TMS32010, 16 bit D/A converter at $120 \mathrm{kHz}$ low pass filtered at $35 \mathrm{kHz}$ ) and presented monaurally via a calibrated headphone (STAX 54) connected to a sound delivery tube sealed into the contralateral ear canal. Characteristic frequency (CF) and threshold were defined audiovisually at all recording locations in all monkeys. Frequency-response areas (FRAs) were defined for all locations in monkeys OM1, OM3, and CM1, and in all three normal monkeys. FRAs were reconstructed from the response to a single stimulus at each of 45 frequencies spanning a $2-5$ octave range centered at the approximate characteristic frequency $(\mathrm{CF})$ and equidistant on a logarithmic scale. Each frequency was presented at each of 15 levels spanning a $70 \mathrm{~dB}$ range in $5 \mathrm{~dB}$ steps (675 total stimuli). Q10dB and Q40dB were defined from these frequency response areas as the CF divided by the bandwidth 
Table 1. Mean and SD of sound intensities (dB SPL) measured at 12 locations within the sound field of the apparatus

\begin{tabular}{lllll} 
Task & S1 stimulus & Below threshold & Near threshold & Above threshold \\
\hline $2.5 \mathrm{kHz}$ & $64.27 \pm 2.7$ & $64.44 \pm 2.89$ & $64.17 \pm 2.93$ & $65.27 \pm 2.93$ \\
$3 \mathrm{kHz}$ & $57.65 \pm 3.97$ & $59.55 \pm 3.01$ & $60.16 \pm 2.90$ & $57.15 \pm 3.78$ \\
$5 \mathrm{kHz}$ & $54.72 \pm 2.93$ & $55.39 \pm 3.08$ & $56.51 \pm 3.47$ & $53.55 \pm 3.88$ \\
$8 \mathrm{kHz}$ & $71.60 \pm 4.69$ & $71.81 \pm 4.35$ & & $73.13 \pm 4.71$
\end{tabular}

Measurements were taken at the mid-range intensity used in the behavioral task. In the $2.5 \mathrm{kHz}, 3 \mathrm{kHz}, 5 \mathrm{kHz}$, and 8 $\mathrm{kHz}$ tasks, the frequencies measured under "Sl stimulus" were $2511 \mathrm{~Hz}, 3188 \mathrm{~Hz}, 4765 \mathrm{~Hz}$, and $7950 \mathrm{~Hz}$; "Below threshold" were $2527 \mathrm{~Hz}, 3275 \mathrm{~Hz}, 4849 \mathrm{~Hz}$, and $8036 \mathrm{~Hz}$; "Near threshold" were $2534 \mathrm{~Hz}, 3367 \mathrm{~Hz}, 4939 \mathrm{~Hz}$; and "Above threshold" were $2613 \mathrm{~Hz}, 3641 \mathrm{~Hz}, 5212 \mathrm{~Hz}$, and $8371 \mathrm{~Hz}$, respectively.

$10 \mathrm{~dB}$ and $40 \mathrm{~dB}$ above minimum threshold, respectively, following methods described by Schreiner and Mendelson (1990) and Sutter and Schreiner (1991). The minimum latency was defined as the time from stimulus onset to the earliest consistent response for all 15 intensity levels presented for the three frequencies nearest CF ( 45 stimuli total).

Electrodes were parylene-insulated tungsten wires with impedances of approximately $1 \mathrm{M} \Omega$ at $1 \mathrm{kHz}$ (Microprobe, Inc). Neural signals were amplified, band-pass filtered (1-10 kHz), and displayed on an oscilloscope and audio amplifier. Spike activity was isolated from this signal with a window discriminator (BAK DIS-1) set to accept all waveforms greater than two times the neural noise level with the time window set for the peak of the largest responses. This eliminated evoked potentials and included approximately one to five independent waveforms of neural origin. The number and arrival time of each event relative to stimulus onset were recorded and stored in a computer (DEC 11/73).

At the start of each experiment, the angle of the electrode was set to be approximately parallel with the angle of the sulcus. Our objective was to define tuning curves limited to neurons in the middle cortical layers, which characteristically have the "best" responses as defined by vigorous activity with a short latency and well-defined tuning curves. Three long penetrations were made approximately $300 \mu \mathrm{m}$ apart and located along a line perpendicular to the sulcus. In these penetrations, the neural response to acoustic stimuli was defined audiovisually in 500 $\mu \mathrm{m}$ steps. By comparing the neural responses at equivalent distances along these three tracks, we were able to verify that the angle of the penetration was roughly parallel to the sulcus. Electrode tracks near the sulcus were continued until an unambiguous border with another cortical field (marked by an abrupt shift in characteristic frequency) was encountered, or until neural activity could no longer be evoked with acoustic stimuli. These penetrations were commonly $3-4.5 \mathrm{~mm}$ in length.

At the conclusion of the electrophysiology experiments, electrolytic lesions were made at selected locations by passing $10 \mu \mathrm{A}$ of direct current for 10-20 sec. Carbon particle marks were made by insertion of a tungsten electrode that had been coated with india ink at four to seven locations surrounding the investigated cortical tissue. The animals were given a lethal injection of barbiturate, and then perfused intracardially with normal saline at $37^{\circ} \mathrm{C}$ followed by one of two fixatives in phosphate buffer: $10 \%$ formalin or $1.5 \%$ paraformaldehyde with $1.5 \%$ glutaraldehyde. Frozen sections were cut at $100 \mu \mathrm{m}$ thickness in the frontal plane. The sections were parallel to the electrode penetrations as indicated by the carbon particle marks. Alternate sections were stained with cresyl violet or for endogenous cytochrome oxidase activity (WongRiley et al., 1978). The angle of the electrode penetrations and the distances between recording locations were reconstructed with reference to the electrolytic lesions and carbon particle marks. AI maps represent reconstructions of all recording locations that corresponded to the middle cortical layers III and IV, approximately 500-900 $\mu \mathrm{m}$ below the pial surface. These maps are presented in two dimensions by projecting the location of the recording site along the radial direction to a point where it intersected an imaginary line drawn $700 \mu \mathrm{m}$ below the cortical surface. This technique limits the distortion that results from projecting recording sites to the surface of the crown of the sulcus. Results from recording locations in other cortical layers are not reported here.

Cortical areas of representation were measured by defining borders as equidistant between neighboring recording locations. The approximate error in these area measurements at interpenetration distances used in these experiments is on the order of 10-20\% (see Merzenich et al., 1987; Recanzone et al., 1992b). Statistical analysis of all behavioral and electrophysiological data was done using the software STATVIEW
$512+$. The specific statistical test was an unpaired one-tailed $t$ test unless otherwise indicated. $p$ values $\leq 0.05$ were considered to be statistically significant.

\section{Results}

\section{Psychophysics}

Improvement in performance with training. The auditory frequency discrimination abilities of each of the five trained monkeys improved progressively with training. The improvement in threshold with training is shown for two representation monkeys in Figure 1. This improvement was characterized by a relatively short and stecp phasc in which large improvements were recorded between sessions, followed by a longer period in which smaller gains were observed. These observations are summarized for each of the five monkeys in Figure 2. The discrimination threshold was averaged over five sessions at different periods roughly equally spaced throughout the several week training period. In almost every case, the largest gains were measured between the initial five sessions and the five sessions taken after approximately one-third of the total number of sessions. The decrease in threshold was generally less throughout the remainder of training, although significant decreases were measured in every monkey $(p<0.05)$. Final mean discrimination thresholds were slightly better for the $2.5 \mathrm{kHz}$ task than the $8 \mathrm{kHz}$ task (see Table 2). By contrast, the final thresholds for the $5 \mathrm{kHz}$ and the $3 \mathrm{kHz}$ tasks in monkeys OM4 and OM 5 were much greater, even though these monkeys also showed significant improvements with training.

Previous studies in owl monkeys on tactile frequency discrimination performance have shown that improvements in minimally detectable frequency differences resulted from (1) a leftward shift in the psychometric function and (2) an increase in the slope of the psychometric function (Recanzone et al., 1992a). Representative psychometric functions from one session within each of the four periods of auditory training shown in Figure 2 are reproduced for monkeys OMl $(A)$ and OM3 $(B)$ in Figure 3. Consistent with the results from the tactile task, the performance at each $S 2$ frequency increased in a roughly sequential fashion, with the performance at large frequency differences improving before the performance at smaller frequency differences. For example, delta frequencies ( $\Delta F$ values) above $300 \mathrm{~Hz}$ for OM 1 had improved to near maximum performance by session 36 , whereas the performance at the $\Delta F$ of $80 \mathrm{~Hz}$ did not begin to improve until after this session. Similar examples could be cited for each monkey.

The slope of the psychometric function also increased throughout the training period. The average slopes of the psychometric function at threshold over five sessions are shown 

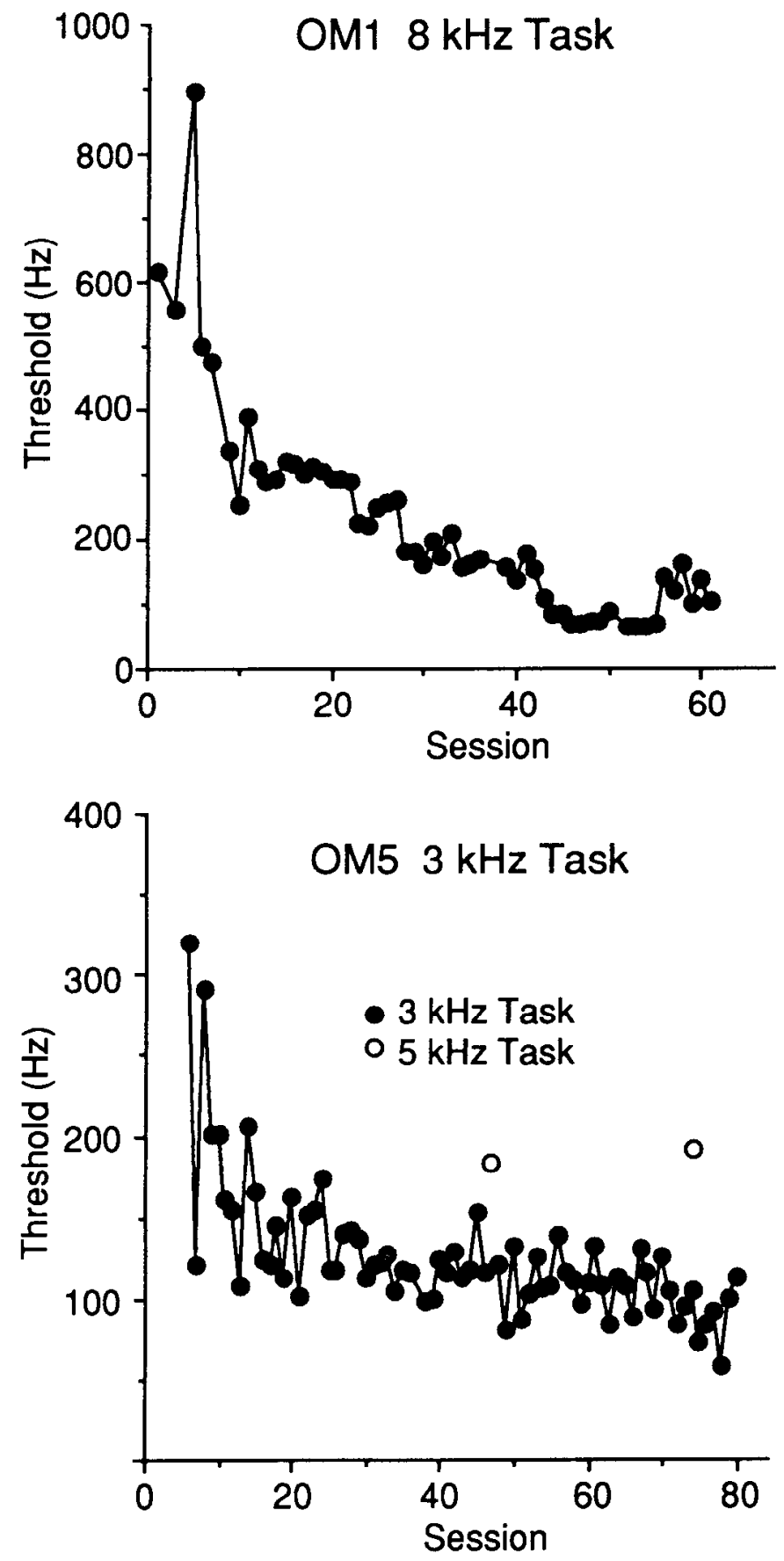

Figure 1. Progressive improvement in the frequency discrimination threshold measured on daily sessions. The delta frequency with a performance of 0.50 is plotted for all sessions in which at least one S2 frequency had a performance below 0.50 (minimum 20 presentations), and the overall false-positive rate was below $15 \%$. Solid circles denote the frequency range tested on repeated sessions $(8 \mathrm{kHz}$ and $3 \mathrm{kHz}$ for $A$ and $B$, respectively), and open circles represent sessions using an untrained frequency range $(5 \mathrm{kHz}$ in $B)$.

for each monkey in Figure 4 for the same training periods as shown in Figure 2. These slopes increased significantly between at least two of the training periods measured, and between the slopes measured in the initial and final training sessions for all monkeys except OM5, which did not reach statistical significance $(p=0.091)$. Thus, consistent with observations in the tactile frequency discrimination task, the improvement in performance was marked by a roughly sequential increase in the

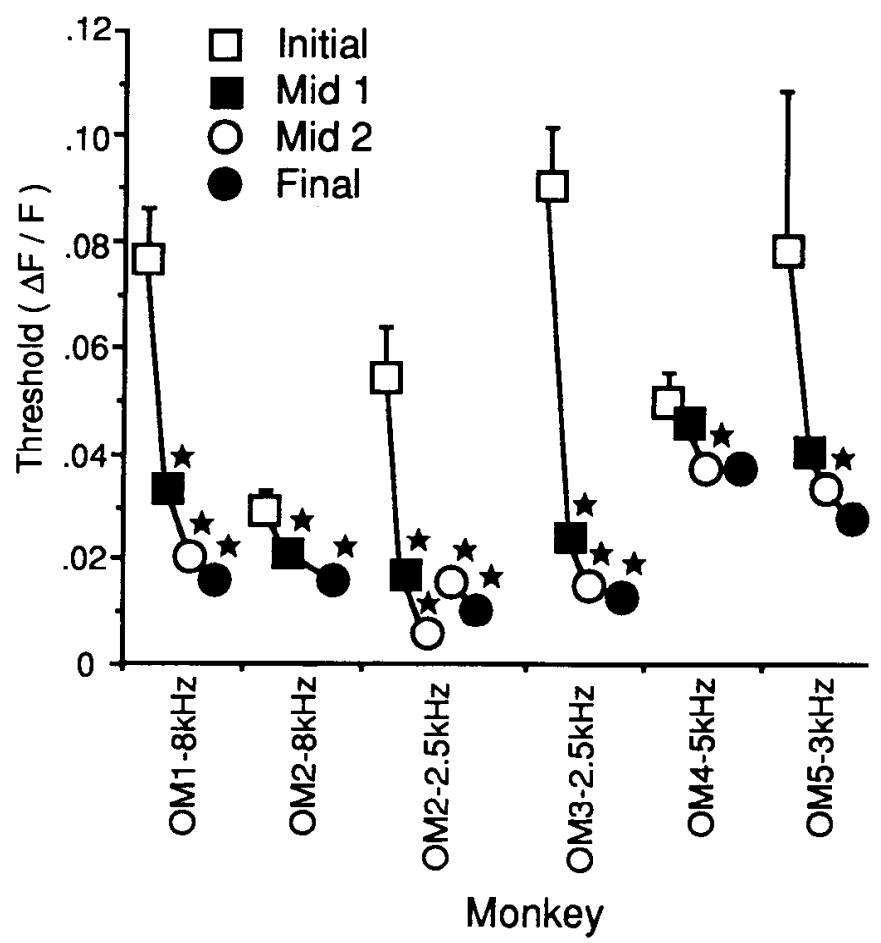

Figure 2. Decrease in frequency discrimination threshold with training. The discrimination thresholds taken over five continuous sessions are shown (mean and SE) for each monkey trained at the auditory frequency discrimination task. Units are given in $\Delta F / F$. Initial sessions are taken as sessions 1-5; Final sessions are the five sessions immediately preceding the electrophysiology experiment. Mid 1 and Mid 2 were chosen to divide the total training period into four roughly equal periods. Error bars not drawn were smaller than the symbol size. Stars indicate statistically significant differences between the starred symbol and the immediately preceding training period. $O M 2-2.5 \mathrm{kHz}$ is shown in two periods because training at the $2.5 \mathrm{kHz}$ task was interrupted by training on the $8 \mathrm{kHz}$ task (see Fig. 6). Sessions shown for this monkey are 1$5,30-34,57-61,88-92$, and $107-111$.

performance at each $\mathrm{S} 2$ frequency and an increase in the slope of the psychometric function at threshold.

One way in which decreases in threshold could occur is by a shift in the monkey's internal criteria by which it makes a re-

Table 2. Final thresholds measured for all behaviorally trained monkeys

\begin{tabular}{llcl} 
Task & Monkey & \# Scssions & $\Delta F / F$ \\
\hline $2.5 \mathrm{kHz}$ & OM2 & $61 / 24$ & $0.005 / 0.010$ \\
$2.5 \mathrm{kHz}$ & OM3 & 97 & 0.013 \\
$3 \mathrm{kHz}$ & OM5 & 80 & 0.028 \\
$3 \mathrm{kHz}$ & OM4 & 6 & 0.045 \\
$5 \mathrm{kHz}$ & OM4 & 75 & 0.038 \\
$5 \mathrm{kHz}$ & OM5 & 2 & 0.040 \\
$8 \mathrm{kHz}$ & OM1 & 61 & 0.015 \\
$8 \mathrm{kHz}$ & OM2 & 26 & 0.016 \\
$8 \mathrm{kHz}$ & OM3 & 4 & 0.041 \\
Passive, $8 \mathrm{kHz}$ & CM1 & 131 & - \\
Passive, $5 \mathrm{kHz}$ & CM2 & 75 & -
\end{tabular}

Threshold measurements represent the mean of the final five sessions for trained tasks, or all sessions for tasks with six or less total training sessions. The two measurements for $\mathrm{OM} 2$ represent the two training periods for the $2.5 \mathrm{kHz}$ task that bracketed the training at the $8 \mathrm{kHz}$ task. No auditory thresholds were derived in the passive-stimulation animals. 

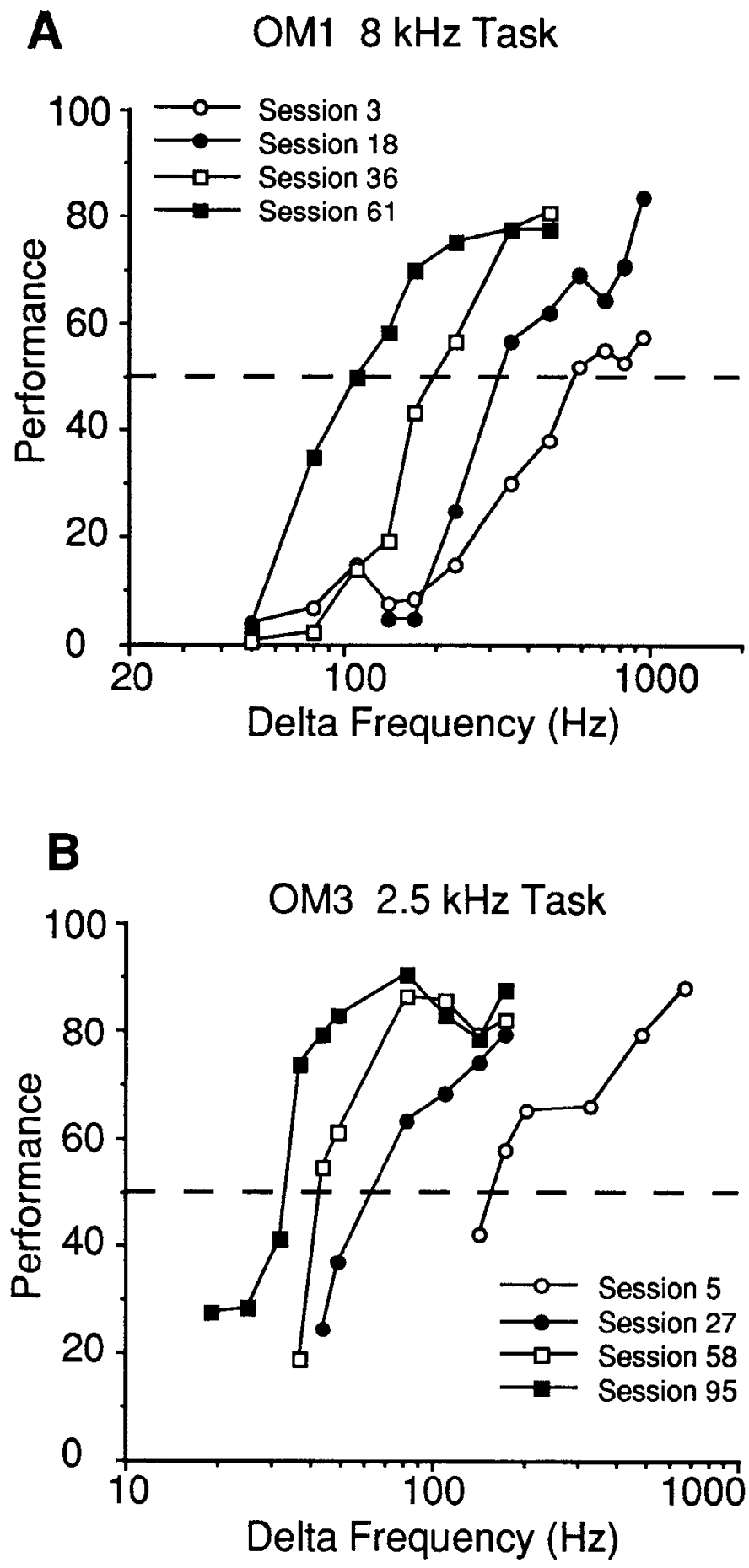

Figure 3. Representative examples of psychometric functions taken during the training periods (see Fig. 2). The broken line defines threshold (50\% performance). Psychometric functions shifted to smaller frequency differences and appeared to become steeper during the course of training in all studied monkeys.

sponse (see Green and Swets, 1966; Recanzone et al., 1992a). The increased slope of the psychometric functions argues against this explanation. A second line of evidence is that the falsepositive rate was maintained below $15 \%$ and did not significantly change over the course of training. Furthermore, there was no significant correlation between the false-positive rate and the hit rate measured for any $\Delta F$ near threshold (hit rates

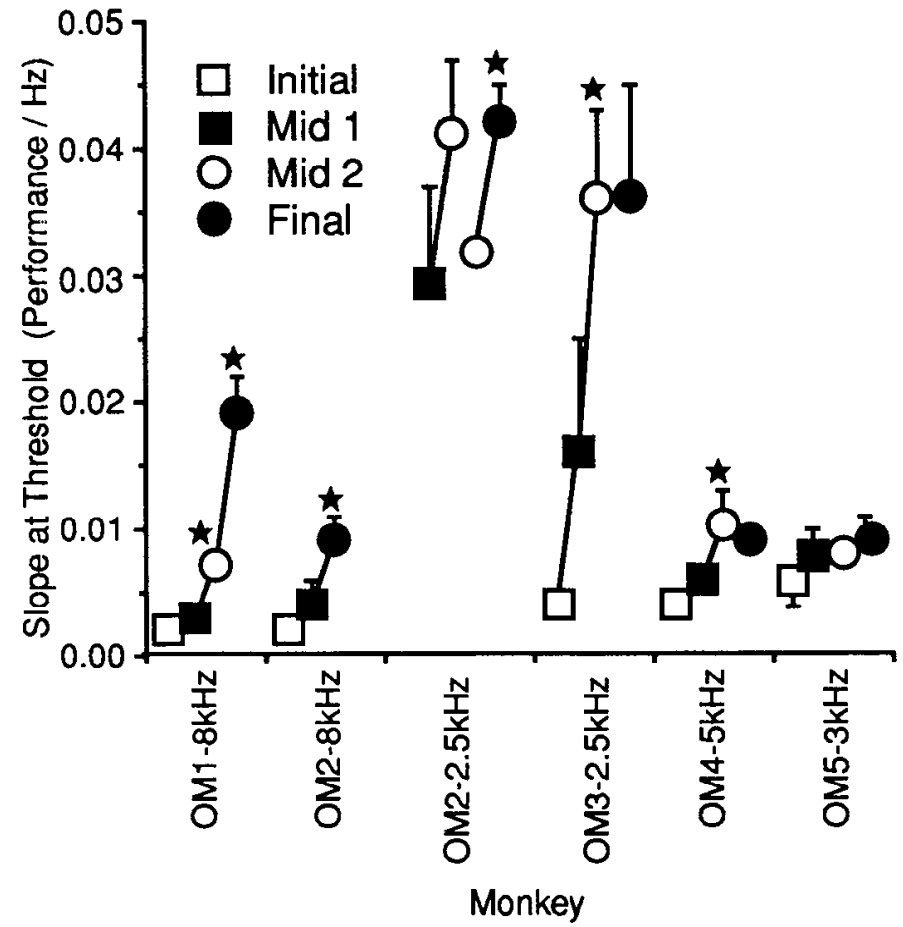

Figure 4. Slope of the psychometric function at threshold in all trained monkeys. The mean and SE of the slope of the psychometric function at threshold are shown for the four training periods as defined in Figure 2 for all monkeys. Stars indicate statistically significant differences.

of 20-90\%) in any of the five owl monkeys studied ( $p>0.05$ in all cases). Finally, the measure of $d^{\prime}$ for at least three $\mathbf{S} 2$ frequencies was analyzed over the four training periods for each monkey. In every monkey there was at least one statistically significant increase in the measure of $d^{\prime}$ between training periods for each frequency tested (and no significant decreases). The results from the two monkeys that had the highest discrimination thresholds are shown in Figure 5. Even in these monkeys, there was a sequential increase in the measure of $d^{\prime}$ with training. These results indicate that the improvements in performance as reflected in the measures of threshold and $d^{\prime}$ reflect a true increase in perceptual acuity with training.

Transference. Previous studies had indicated that training one sensory surface in a perceptual task confers limited gains in that task to nearby sensory surfaces (see James, 1890; Craig, 1988; Recanzone et al., 1992a). In each monkey excluding OM1, the performance at one other frequency range was tested at various times throughout the training period (Table 2). The most extensively studied animal was OM2, which was trained initially on the $2.5 \mathrm{kHz}$ task, was then shifted to the $8 \mathrm{kHz}$ task, and was finally returned to the $2.5 \mathrm{kHz}$ task (Fig. 6). The characteristic decrease in threshold was observed for both the $2.5 \mathrm{kHz}$ task (solid circles) and the $8 \mathrm{kHz}$ task (open circles). The thresholds measured on the $2.5 \mathrm{kHz}$ task increased during the course of training at the $8 \mathrm{kHz}$ task. The thresholds then decreased again when the task was shifted back to $2.5 \mathrm{kHz}$. A second feature to note is that the $8 \mathrm{kHz}$ task does not show a rapid improvement in performance at the initial period of training (compare Fig. 6 with Fig. 1, and see Fig. 2).

If a particular frequency outside the normal training range was tested on two or three individual sessions that were widely spaced in time, there was no improvement in performance for 


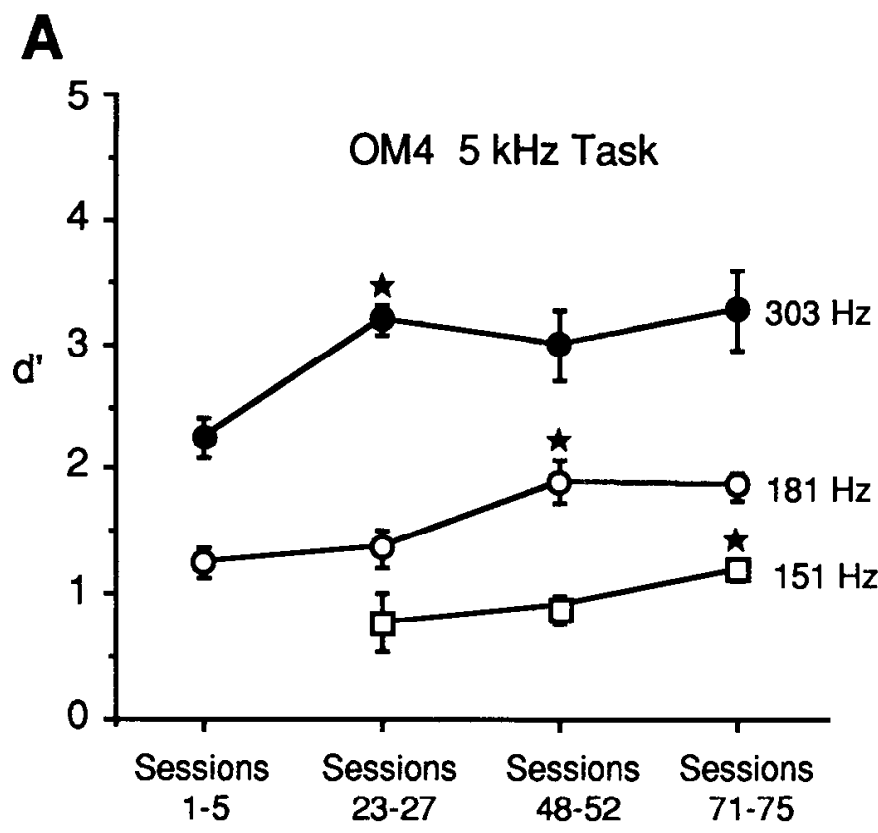

B

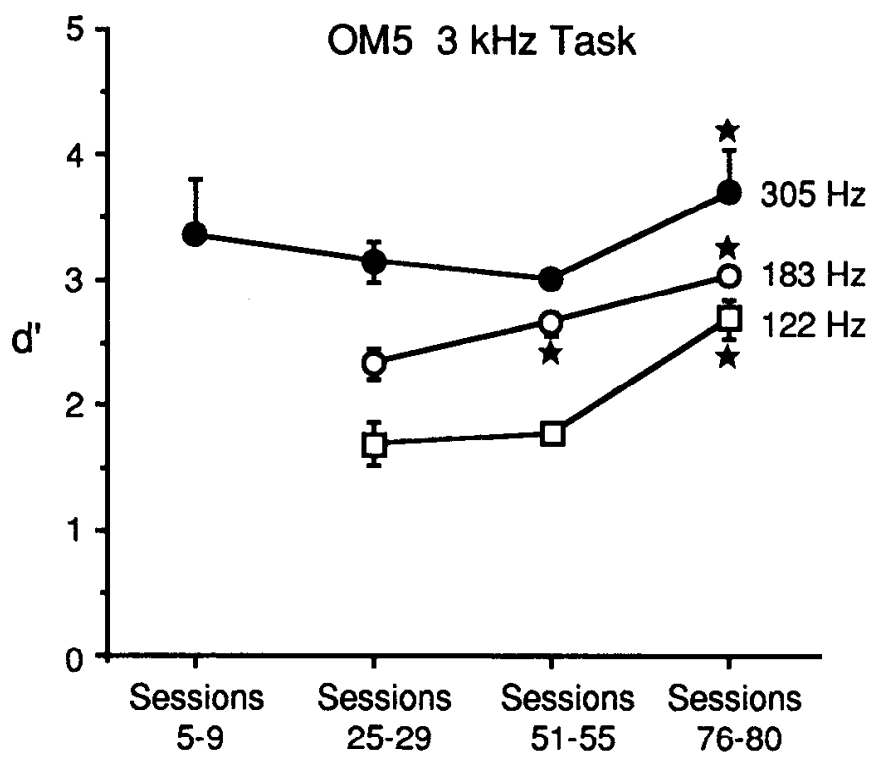

Figure 5. Measures of $d^{\prime}$ for three frequencies as a function of training. The points for a single frequency are connected by a solid line. Error bars indicate SE; stars indicate statistically significant differences from the preceding measure.

that frequency. This is shown by the performance at $5 \mathrm{kHz}$ in a monkey trained at the $3 \mathrm{kHz}$ task (OM5; open circles, Fig. $1 B$ ), and by the psychometric functions derived at $3 \mathrm{kHz}$ for a monkey trained at the $5 \mathrm{kHz}$ task (OM4; Fig. 7). In every example, even though there was a significant improvement between these sessions for the trained frequencies, there was little difference in the performance at frequencies that were not trained.

Essentially all training was conducted with the $\mathrm{S} 2$ frequencies above the $S 1$ frequency $(+\Delta F)$. A somewhat different effect was observed by occasionally testing the performance with decreased $\mathrm{S} 2$ frequencies $(-\Delta F \mathrm{~s})$. On three sessions, OM4 was presented with S2 stimuli in which the second tone of the pair was lower

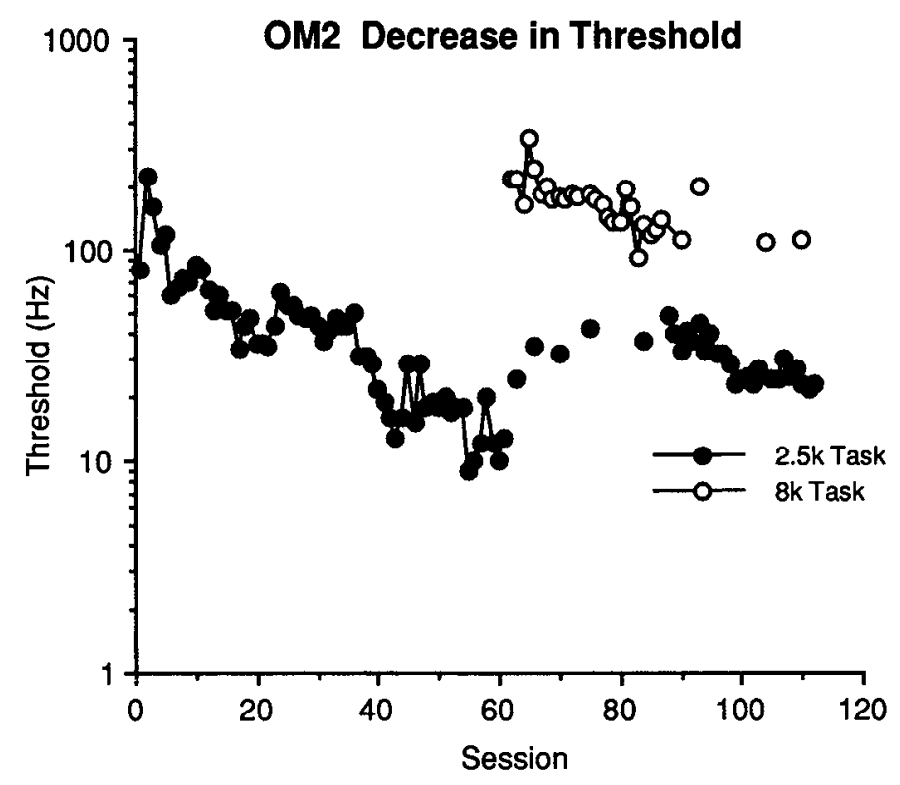

Figure 6. Improvement in frequency discrimination threshold at two different $\mathrm{S} 1$ frequencies for monkey OM2. OM2 was initially trained at the $2.5 \mathrm{kHz}$ task (solid circles), then changed to the $8 \mathrm{kHz}$ task (open circles), and finally returned to the $2.5 \mathrm{kHz}$ task. The $2.5 \mathrm{kHz}$ task was tested intermittently during the consecutive training sessions at the 8 $\mathrm{kHz}$ task and vice versa.

in frequency than the first. The psychometric functions from these three sessions are shown in Figure 8 (left), along with three functions using an increased frequency $\mathrm{S} 2$ stimulus $(+\Delta F$, right $)$ taken at a similar period of training in the same monkey. The

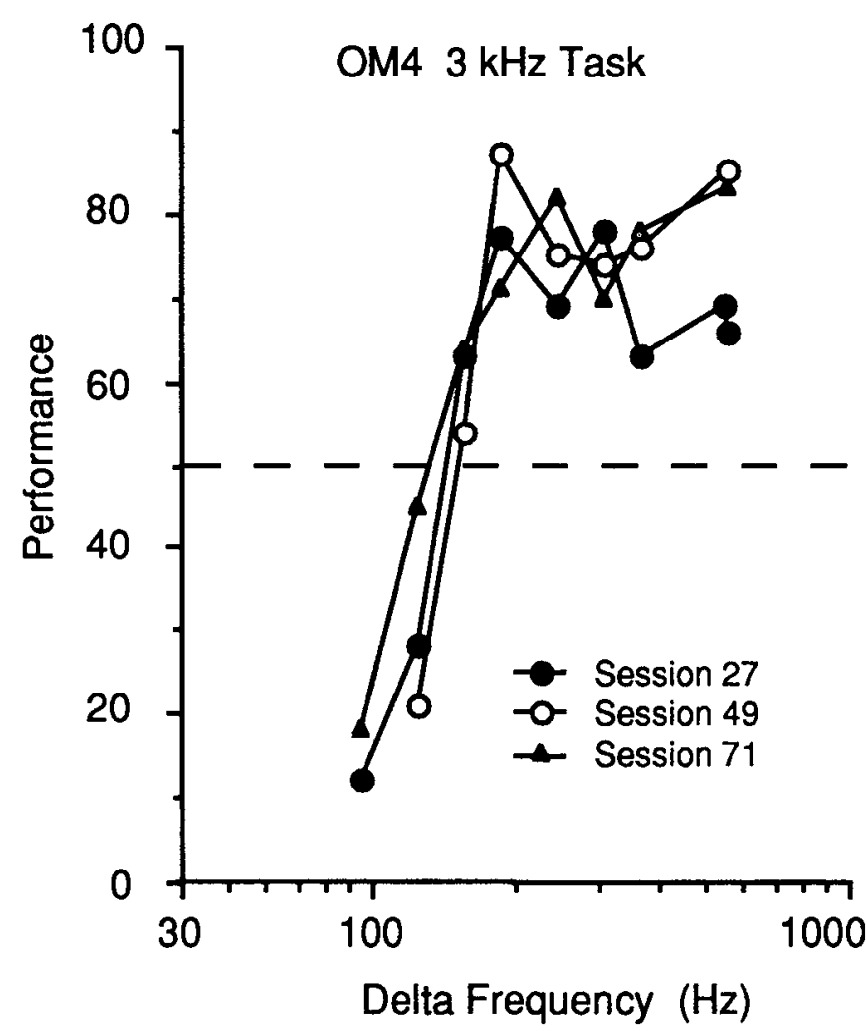

Figure 7. Psychometric functions at three $3 \mathrm{kHz}$ task testing sessions for the monkey trained on the $5 \mathrm{kHz}$ task (OM4). The broken line represents performance of $50 \%$ (threshold). 


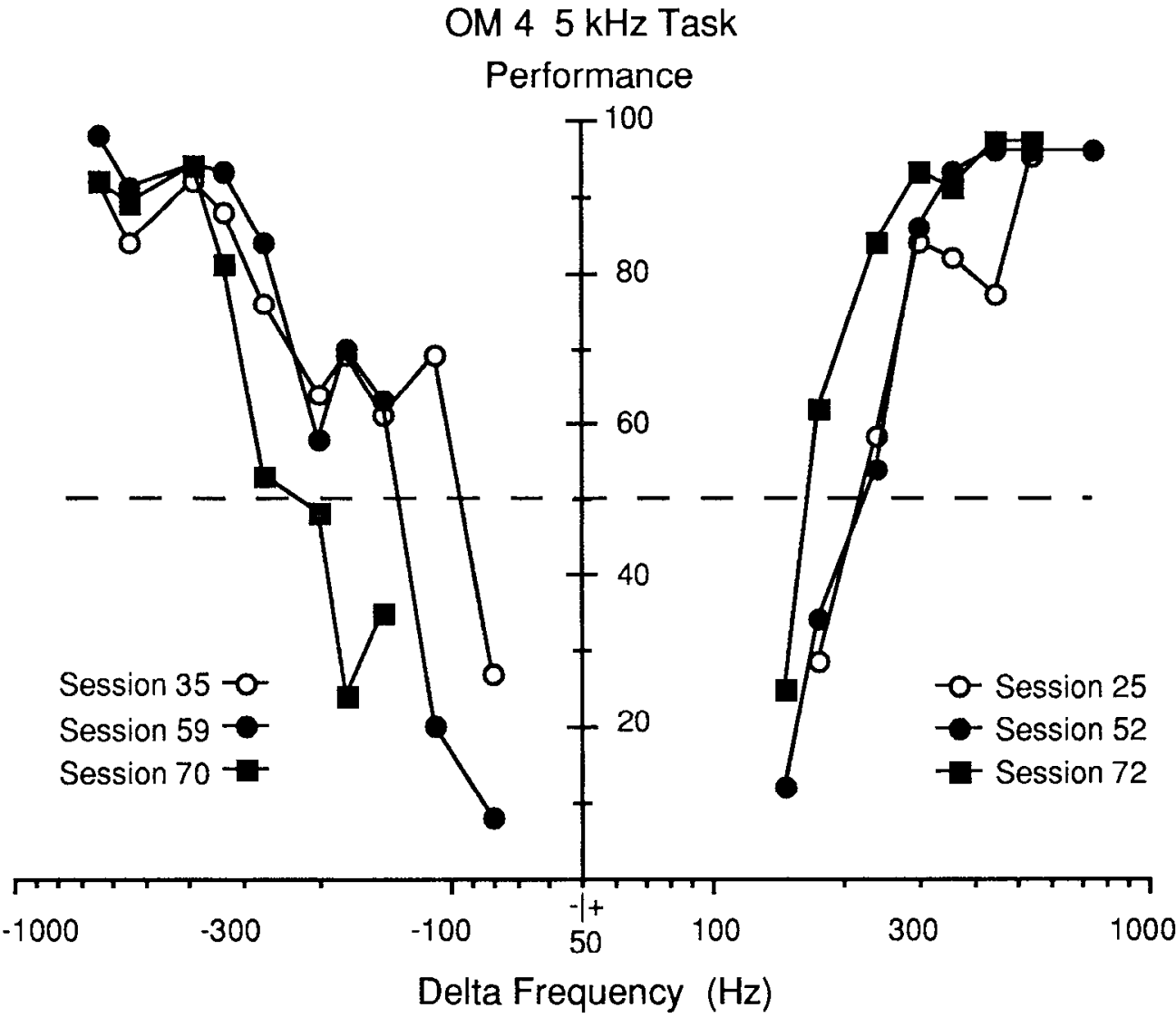

Figure 8. Representative psychometric functions for $+\Delta F$ (right) and $-\Delta F$ (left) tasks for the $5 \mathrm{kHz}$ standard for monkey OM4. The y-axis intercepts the delta frequency of $\pm 50 \mathrm{~Hz}$. Note the increase in performance for the $+\Delta F$ task and the decrease of performance for the $-\Delta F$ task with training. functions for the $+\Delta F$ values show the improvement in performance as seen in other monkeys. The functions for $-\Delta F$ values, however, show a worsening of performance and an increase in threshold as a function of session. The worst performance at the $-\Delta F$ task was seen on session 70 , in which there were 10 intervening sessions using the increasing delta frequencies. This greatest decrease in performance coincides with an increase in the performance for the $+\Delta F$ stimuli. The same test on the $3 \mathrm{kHz}$ task was done in monkey OM5 near the end of the training period, and the threshold for the $-\Delta F$ task $(\Delta F / F$ $=0.035)$ was greater than that observed for the $+\Delta F \operatorname{task}(\Delta F / F$ $=0.028$ ).

Effect of stimulus intensity. The effects of transference for other frequencies raise the question of how the performance would be affected for other intensities since the training occurred within a limited intensity range of only 10-12 dB. To address this question, psychometric functions were derived at different intensities in monkeys OM4 and OM5 near the end of the training period. There was a slight decrease in frequency discrimination thresholds with increases in intensity (Fig. 9), but these two parameters were not correlated $(p>0.15$ in both cases).

\section{Electrophysiology}

Functional organization of $A I$. Basic aspects of the functional organization of AI were determined for both the left and right hemispheres in behaviorally trained monkey OMl and in one hemisphere of monkeys OM3 and OM4. Sufficient electrophysiological data were not obtained from trained monkeys OM2 and OM5 and will not be discussed further. AI was also defined in the two passively stimulated control monkeys CM1 and CM2, and in three behaviorally naive owl monkeys (N1, $\mathrm{N} 2$, and N3). Neural responses in laminae III and IV of AI were marked by short-latency (approximately 10-12 $\mathrm{msec}$ ) responses to a relatively narrow band of frequencies. In general, the low

\section{Effects of Intensity on Threshold}

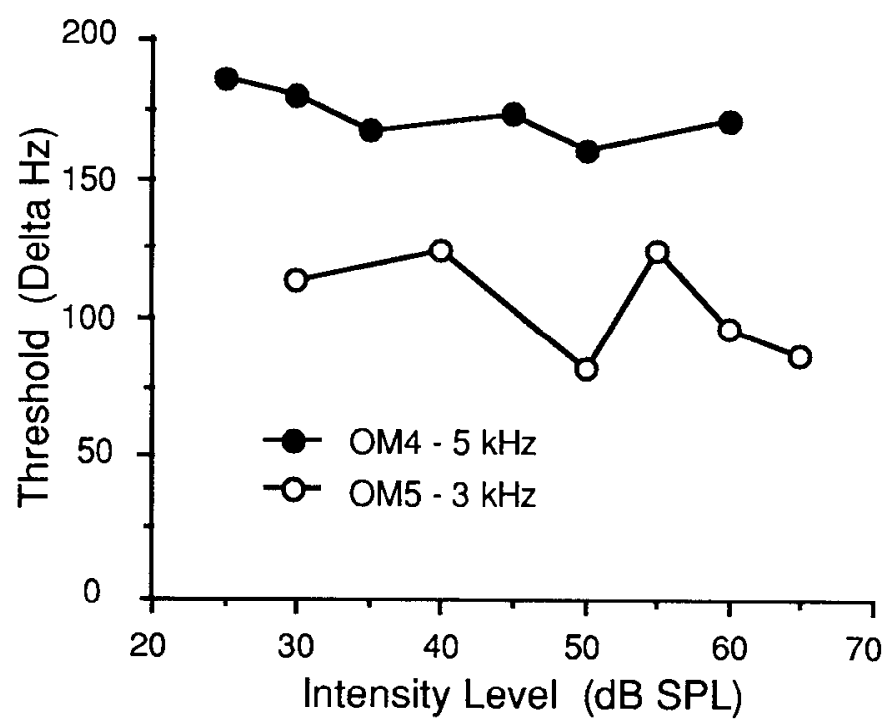

Figure 9. Threshold measured at the trained frequencies as a function of stimulus level for two monkeys, OM4 (solid circles) and OM5 (open circles). All data were taken from single sessions. The intensity represents the mean intensity measured in the sound field occupied by the monkey's head (see Materials and Methods). Stimulus level varied independently for each tone pip over a range of approximately $15-20 \%$ of the mean value plotted. 
frequencies were represented on the crown of the gyrus and the higher frequencies were represented down the lateral bank of the sulcus. Neural responses were clustered into "isofrequency bands" where all neurons had similar CFs. These bands were usually oriented oblique to the sulcus but could also be roughly parallel to it. Within the depths of the sulcus, the neural responses would either become very broadly tuned with a higher threshold and longer latency, or become unresponsive to acoustic stimulation. These regions corresponded with the cytoarchitectonic borders of AI as seen histologically, and were characteristic of other auditory cortical fields in this primate species (see Imig et al., 1977; Merzenich et al., 1991).

A central finding of these studies is that the number of recording locations and the cortical area of representation for the frequencies used in the behavioral task were both increased when compared to those frequencies not used in the training. The reconstructed frequency representations from four owl monkeys are shown in Figure 10. Recording locations at which the $\mathrm{CF}$ was within the range of frequencies presented in the behavioral tasks are indicated by frequency-specific shading. The total cortical arca representing these frequency ranges varied from animal to animal. Some but not all of the differences appeared to be related to the training task. A given frequency was usually represented in a relatively restricted region, but not necessarily continuously. For example, the representation of the frequencies used in the $2.5 \mathrm{kHz}$ task occupied several patches in the trained monkey OM3. By contrast, only a few locations represented these specific frequencies in monkeys not trained at that frequency. Similarly, the greatest representation of the frequencies used in the $5 \mathrm{kHz}$ task were seen in OM4, even though there was only a limited improvement in performance and a relatively small cortical area of representation (Fig. 10D). This effect was not seen in the passively stimulated monkey CM2, which received the same acoustic stimulation as OM4, where only a single cortical location represented the frequencies used in the $5 \mathrm{kHz}$ task at CF (Fig. 10C). No more than two locations with a $\mathrm{CF}$ of $5 \mathrm{kHz}$ were encountered in any of the other monkeys.

The tuning curve data are summarized for all monkeys by plotting the number of cortical locations having a CF within a $500 \mathrm{~Hz}$ range (Fig. 11). In Figure 11, the bin size was adjusted to include all the frequencies used in the behavioral tasks (indicated by arrows). In normal owl monkeys, even though all frequencies are represented, there is a consistent underrepresentation of frequencies between 3 and $8 \mathrm{kHz}$ (Fig. 11A) and most monkeys showed a relatively large number of recording locations with CFs above $15 \mathrm{kHz}$. These monkeys also had a large number of locations with a $\mathrm{CF}$ near $2 \mathrm{kHz}$, but those CFs were not in the range used in the $2.5 \mathrm{kHz}$ behavioral task. Each individual monkey showed idiosyncratic increases of particular frequencies, for example, approximately $10 \mathrm{kHz}$ for monkey $\mathrm{N} 2$, or approximately $2 \mathrm{kHz}$ for monkey $\mathrm{N} 3$.

By contrast, the numbers of locations with CFs in the range of frequencies used in the behavioral task were greatest in monkeys trained at that frequency range. In the trained monkey, the number of locations near $8 \mathrm{kHz}$ was approximately 3-10 times greater in both hemispheres compared to normal and control monkeys (Fig. $11 B$ ). Monkey $\mathrm{CM} 1$, which received the same acoustic stimuli as OM1 but was not required to attend to these stimuli, had an essentially normal representation of these frequencies. The frequency range used in the $2.5 \mathrm{kHz}$ task was represented by more locations in the monkey trained at that task than in any other monkey. The same was true for the frequency range used in the $5 \mathrm{kHz}$ task (Fig. $1 \mathrm{lC}$ ).

The larger number of cortical locations that represented the trained frequencies could not be explained by an increase in the sampling density over that region, as the representation of these frequencies and surrounding areas was defined at equivalent density in all monkeys. This increase was better explained by an increase in the cortical area of representation of those frequencies. Figure 12 shows the cortical areas associated with each of the three different frequency ranges for all monkeys. In every case, the area of representation for the behaviorally relevant frequencies for a trained monkey (solid bars) was significantly greater than the cortical areas for those frequencies in all other monkeys, including those that were passively stimulated (hatched bars).

Response properties at individual locations. The tuning properties of the neurons at each recording location were investigated by recording FRAs at all AI recording locations of monkeys OMl, OM3, CMl, and in each of the three normal monkeys. Measures of Q10dB were made at each location; examples are plotted in Figure 13. In every monkey, the values for Q10dB tended to increase with increasing frequency, consistent with reports from other species (see Pelleg-Toiba and Wollberg, 1989; Schreiner and Mendelson, 1990). There was a considerable range of $Q 10 \mathrm{~dB}$ values for any given frequency in all monkeys. The Q10dB measures for neurons in trained monkeys were greater (tuning bandwidths were narrower) for the behaviorally trained frequencies when compared to normal or passive stimulation control monkeys. The mean Q10dB and Q40dB for all monkeys over the frequency range used in the 2.5 or $8 \mathrm{kHz}$ tasks are shown in Figure 14A. The left and right hemispheres of OM1 are considered independently, and the data from the three normal monkeys are pooled. Measures of Q10dB were statistically significantly greater over the behaviorally trained frequencies in trained monkeys when compared to the three normal controls. The $\mathrm{Q} 10 \mathrm{~dB}$ of the passive control animals was also elevated over that of normal control monkeys. Statistically significant increases in $\mathrm{Q} 40 \mathrm{~dB}$ were noted only in the right hemisphere of OM1 for the $8 \mathrm{kHz}$ frequencies.

In addition to the increase in $\mathrm{Q} 10 \mathrm{~dB}$, there was also an increase in the minimum latency of the response for the behaviorally trained hemispheres in each of the three cases (Fig. 14B). Response latencies in the passive stimulation monkey were not significantly different from those in control monkeys. The variance of the latencies was very low in all monkeys, as indicated by the small SE bars. The distribution of the variance in the latency measure was not statistically significantly different between the normal monkeys and any others $(p>0.1)$.

Correlation of behavioral performance with neural responses. The main objective of these studies was to determine (1) if the functional organization of the primary auditory cortex was altered consequent to training the animal in an acoustic discrimination task, and (2) if the resulting cortical representations were correlated with the behavioral performance. The three measures showing significant changes in trained monkeys described above, the increased cortical area, increased Q10dB (narrower bandwidths near threshold), and increased latency, were subjected to regression analysis. For these analyses, behavioral performance was taken as the threshold measured at the end of the training sessions for the trained monkeys, and paired with the electrophysiological measures derived from those same monkeys. To estimate the behavioral performance of "untrained" 
A Normal Owl Monkey

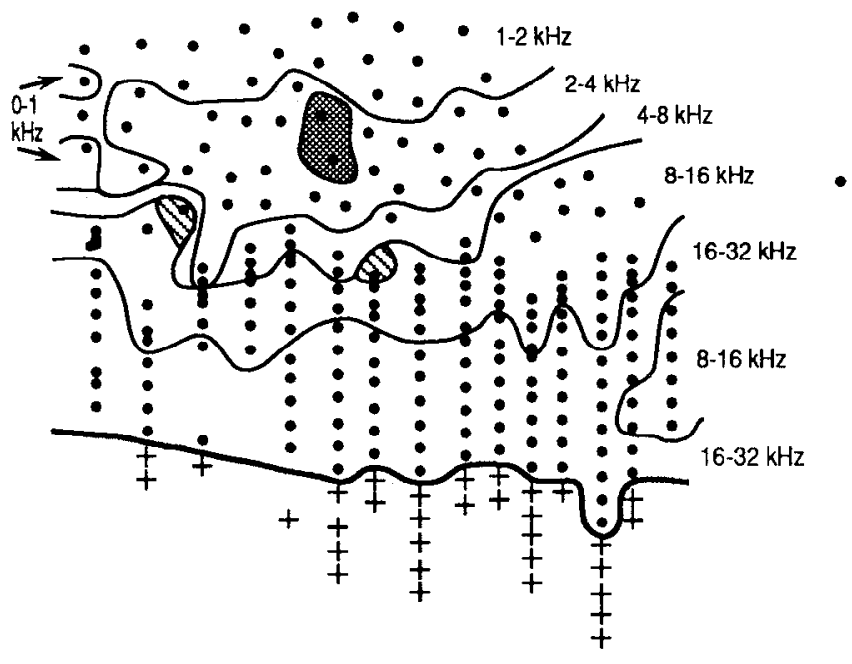

B OM3 Trained on $2.5 \mathrm{kHz}$ Task

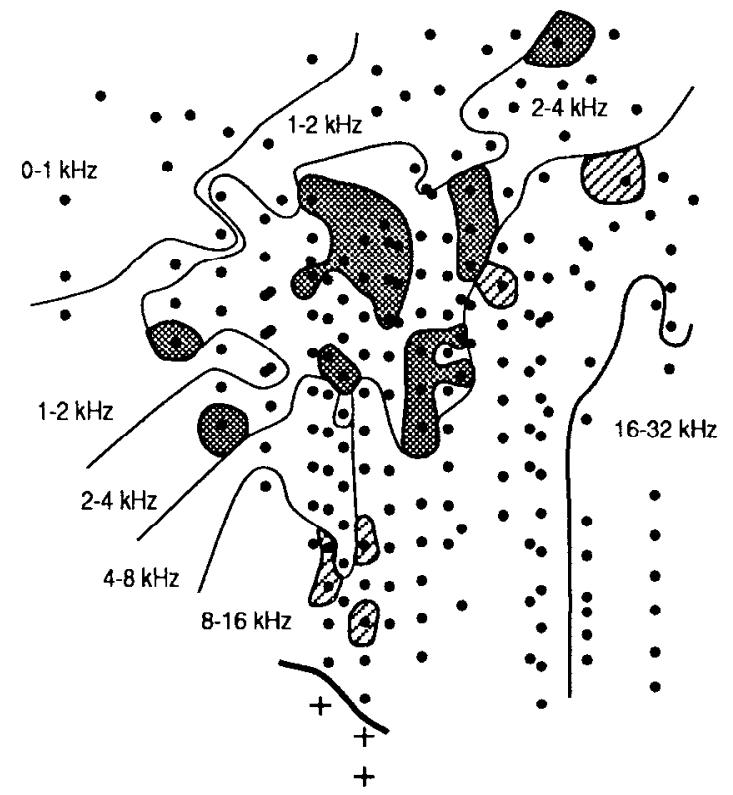

\section{C $\quad$ CM2 Passive Stimulation at $5 \mathrm{kHz}$}

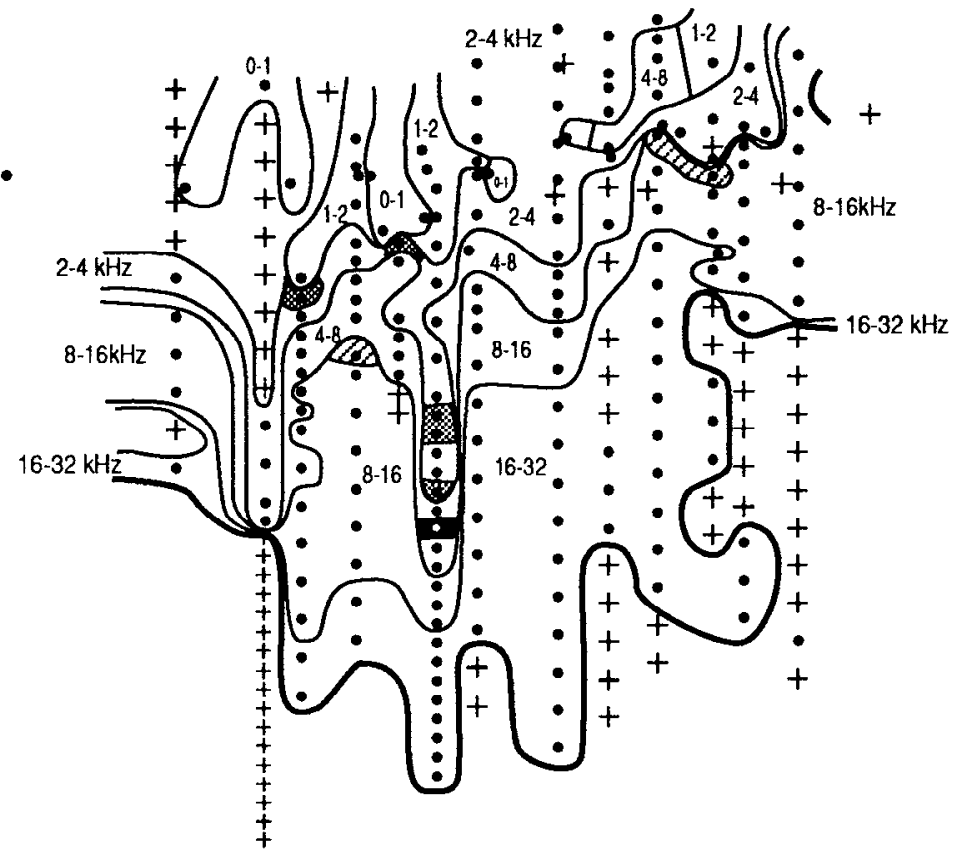

D OM4 Trained on $5 \mathrm{kHz}$ Task
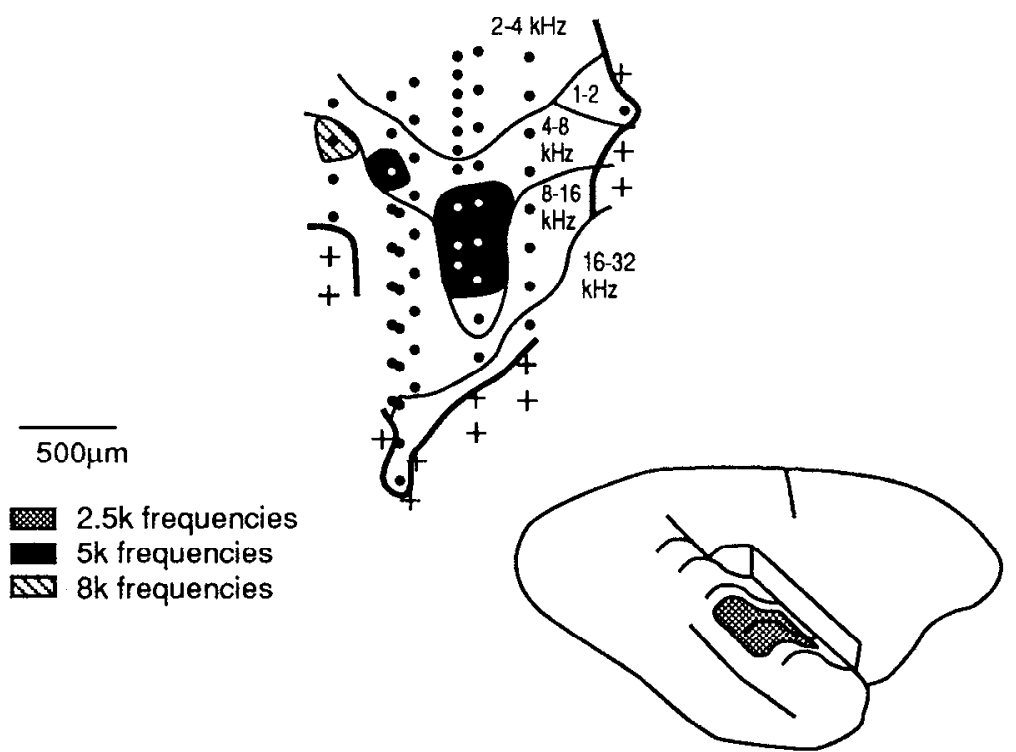

Figure 10. Cortical representations of $\mathrm{CF}$ in $\mathrm{Al}$ of four adult owl monkeys. Thin lines define boundaries of cortical locations with $\mathrm{CFs}$ within one octave. Stippled regions encircle cortical locations where neurons were recorded with CFs in the frequency range used in the $2.5 \mathrm{kHz}$ task, solid regions represent frequencies used in the $5 \mathrm{kHz}$ task, and hatched regions represent the frequency range used in the $8 \mathrm{kHz}$ task. The cortical areas representing a given frequency range were approximated by connecting the $50 \%$ distance values to the neighboring recording sites with CFs outside the given frequency range. Pluses denote recording sites with neuronal responses not consistent with properties of AI neurons. $A$ is from a representative normal owl monkey (N2), $B$ is from a monkey trained at $2.5 \mathrm{kHz}(\mathrm{OM} 3)$, $C$ shows the monkey passively stimulated with the frequencies used in the $5 \mathrm{kHz}$ task (CM2), and $D$ shows the representation of the monkey trained at the $5 \mathrm{kHz}$ task (OM4).

monkeys, the mean threshold of training sessions 5-10 for monkeys OM 3 and $\mathrm{OMI}$ were used for the $2.5 \mathrm{kHz}$ and $8 \mathrm{kHz}$ tasks, respectively. These sessions were used because they showed less variability than the initial five sessions (1-5) and probably better reflect the monkey's true initial discrimination abilities (see Discussion). For the "untrained" behavioral performance of the 3
$\mathrm{kHz}$ and $5 \mathrm{kHz}$ tasks, the mean thresholds for the two and six sessions at these tasks for monkeys OM4 and OM5 were used, respectively. These "untrained" behavioral measures were considered with the electrophysiological results pooled from the untrained monkeys. The values of $\mathrm{Q} 10 \mathrm{~dB}, \mathrm{Q} 40 \mathrm{~dB}$, and minimum latency showed no significant correlation with these be- 


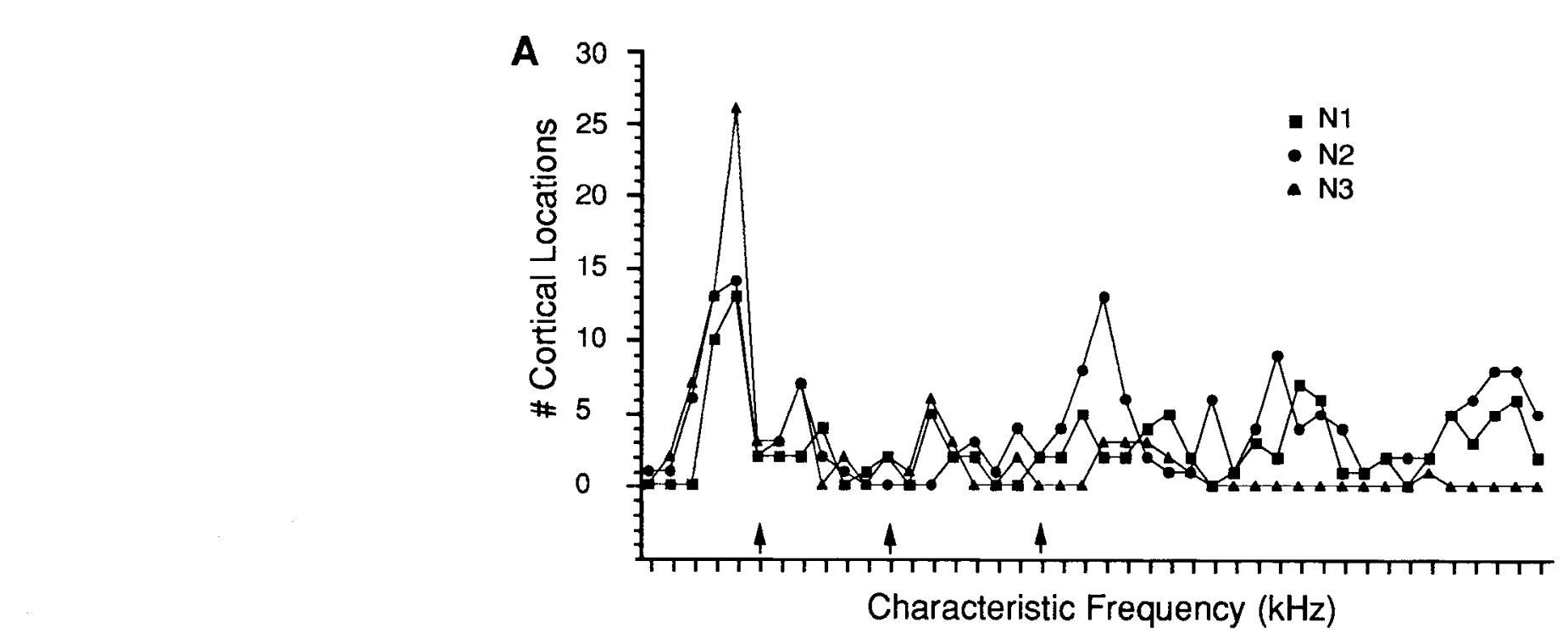

Figure 11. The number of cortical locations plotted by CF in $500 \mathrm{~Hz}$ bins. Bin size was adjusted to incorporate all frequencies used in the behavioral task into a single bin (indicated by arrows). The three normal monkeys are shown in $A$, the two hemispheres from monkey $\mathrm{OM} 1$ and the passive-stimulation control monkey CM1 are shown in $B$, and the monkeys trained at the $5 \mathrm{kHz}$ and $2.5 \mathrm{kHz}$ task (OM4 and OM3) and the passively stimulated monkey CM2 are

B
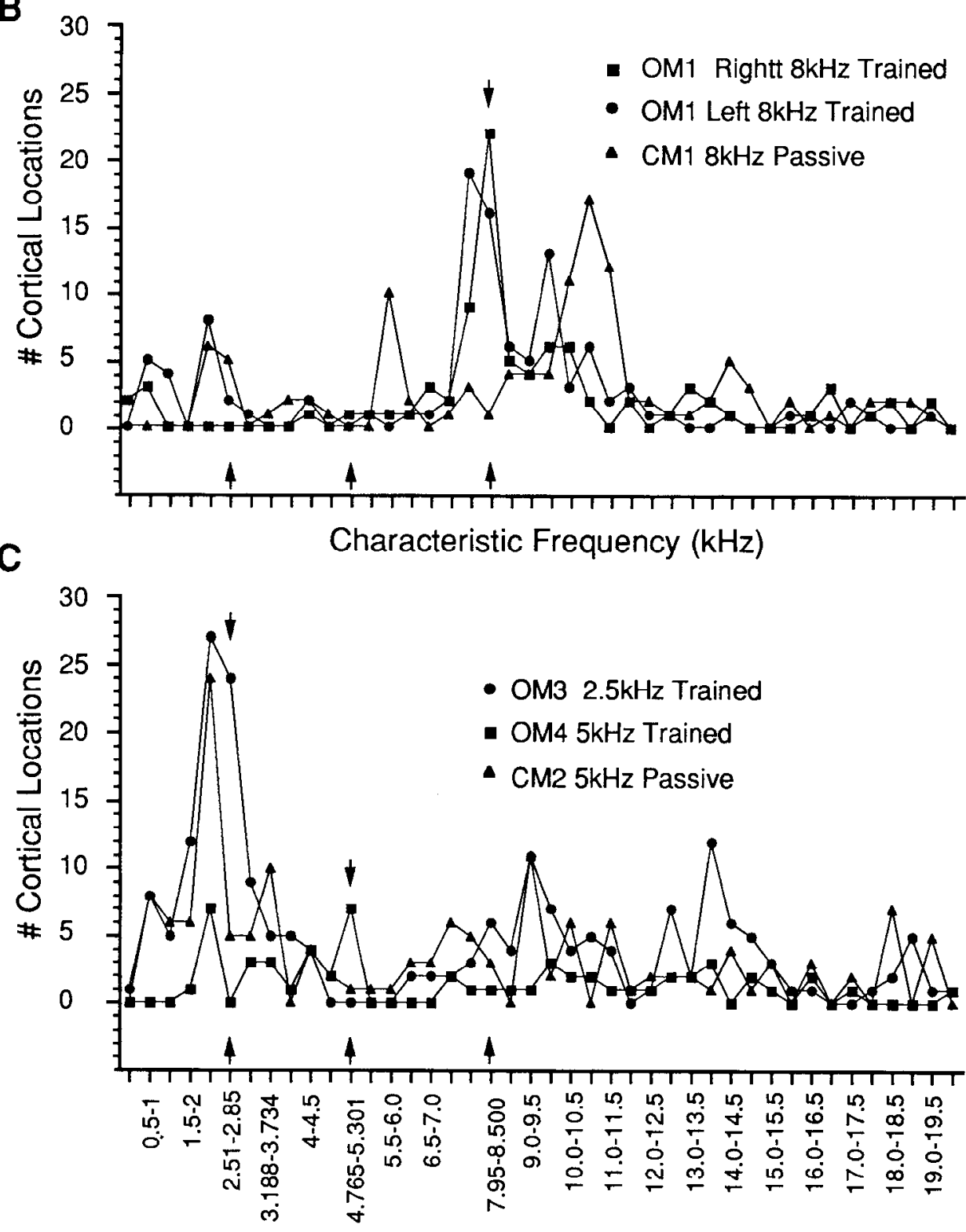
shown in $C$. 

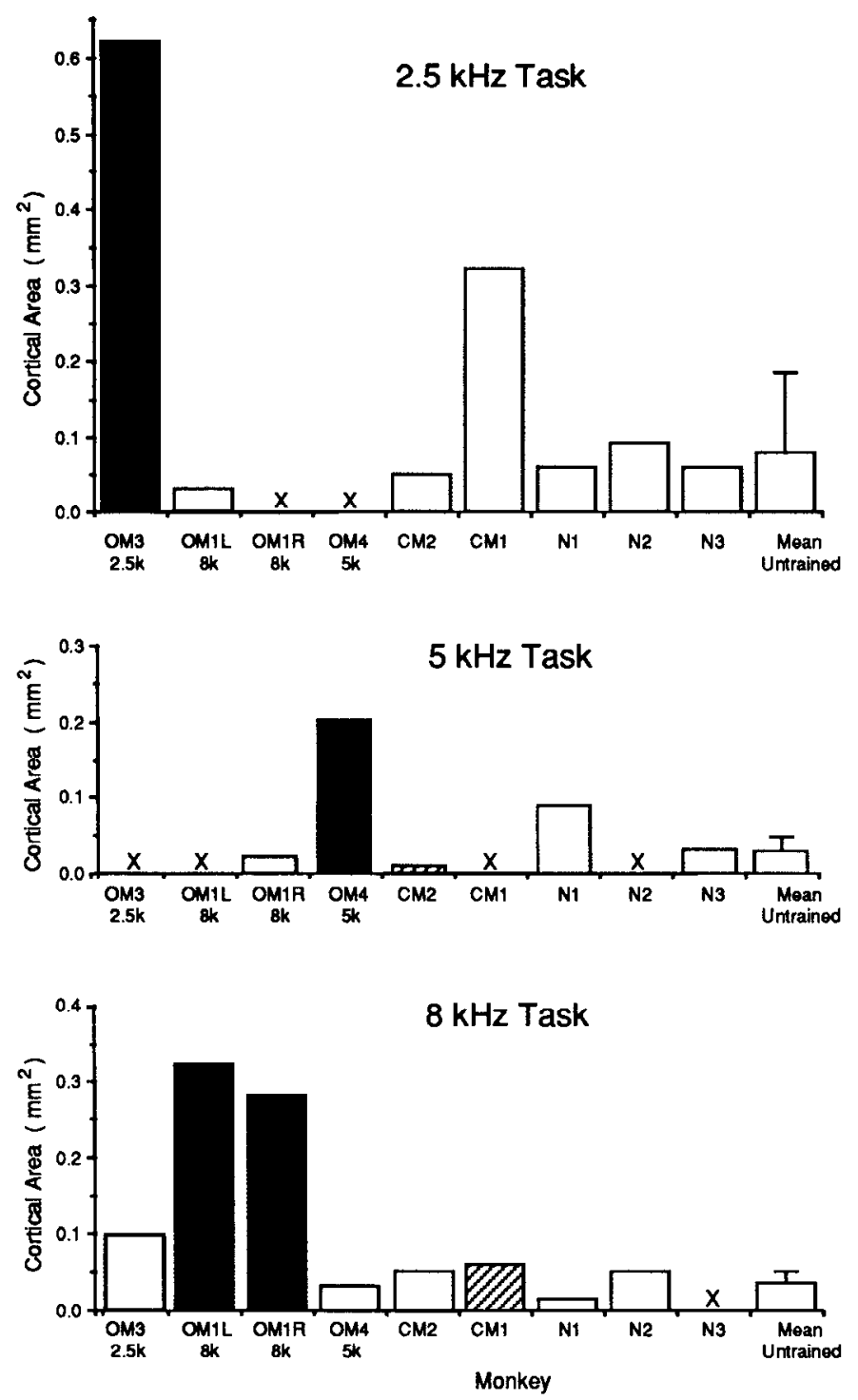

Figure 12. Cortical area of representation of the frequency ranges used in the behavioral study. Solid bars indicate the area of representation of the trained frequency for that particular monkey; hatched bars represent areas of the frequencies presented to the passive-stimulation control monkeys. Crosses represent hemispheres where no cortical locations were recorded with a $C F$ within that frequency range. The column "Mean Untrained" represents the mean and SD for all hemispheres excluding the trained hemispheres.

havioral measures ( $p=0.44,0.21$, and 0.91 , respectively). By contrast, the correlation analysis of behavioral threshold with cortical area revealed that these two parameters were significantly correlated (Fig. 15; $r=0.88 ; p<0.01 ; \mathrm{df}=6$ ).

\section{Discussion}

Improvements in behavioral performance in an auditory frequency discrimination task were recorded in five adult owl monkeys. There was a roughly sequential improvement in performance at individual S2 frequencies, which was expressed in a shift of the psychometric function and an increase in the slope of the function near threshold. The improvement in performance was much less for untrained frequency ranges, and the performance was independent of stimulus level between 25 and $65 \mathrm{~dB}$ SPI. Electrophysiological mapping experiments revealed
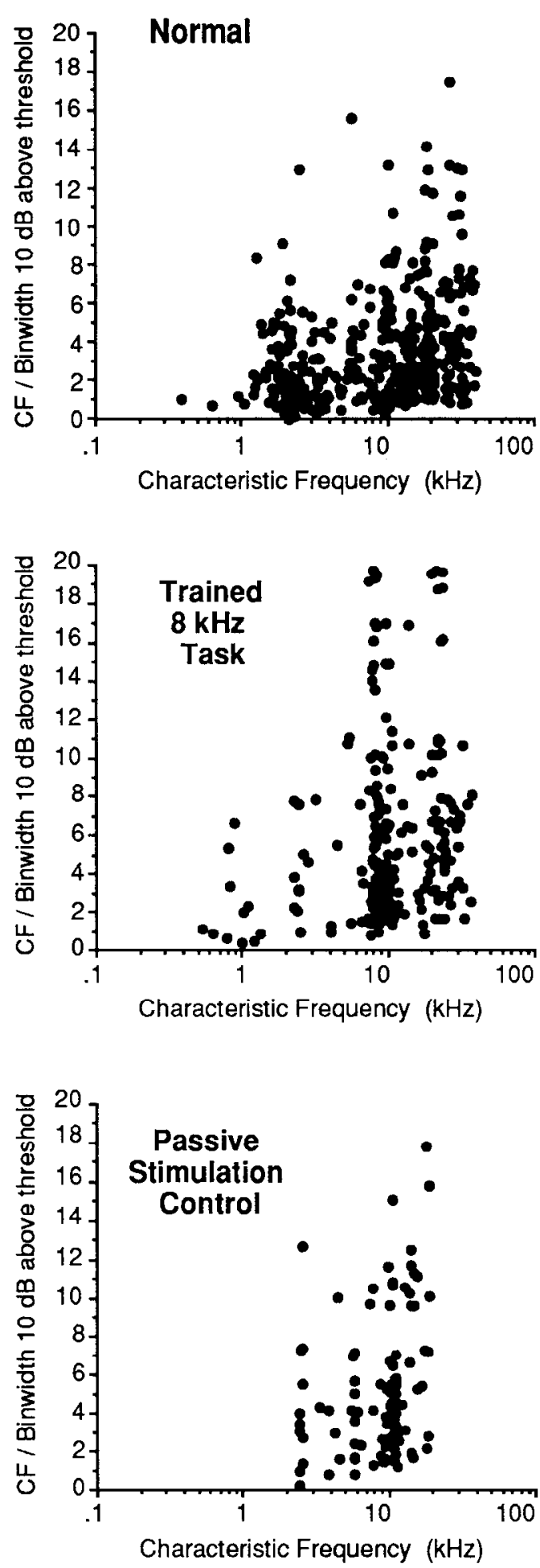

Figure 13. Values of Q10dB as a function of CF. Normal owl monkeys $\mathrm{N} 1, \mathrm{~N} 2$, and $\mathrm{N} 3$ are pooled and shown in the top panel, both hemispheres of trained monkey OM1 in the middle panel, and the passive stimulation control monkey CM1 in the bottom panel. Each point represents the $\mathrm{CF}$ and $\mathrm{Q} 10 \mathrm{~dB}$ at a single recording location.

a larger cortical area of representation of the behaviorally trained frequencies, sharper tuning as measured by Q10dB but not $\mathrm{Q} 40 \mathrm{~dB}$, and longer response latencies over the frequency range used in the behavioral task. The cortical area of CF representation was the only measured parameter that was significantly 


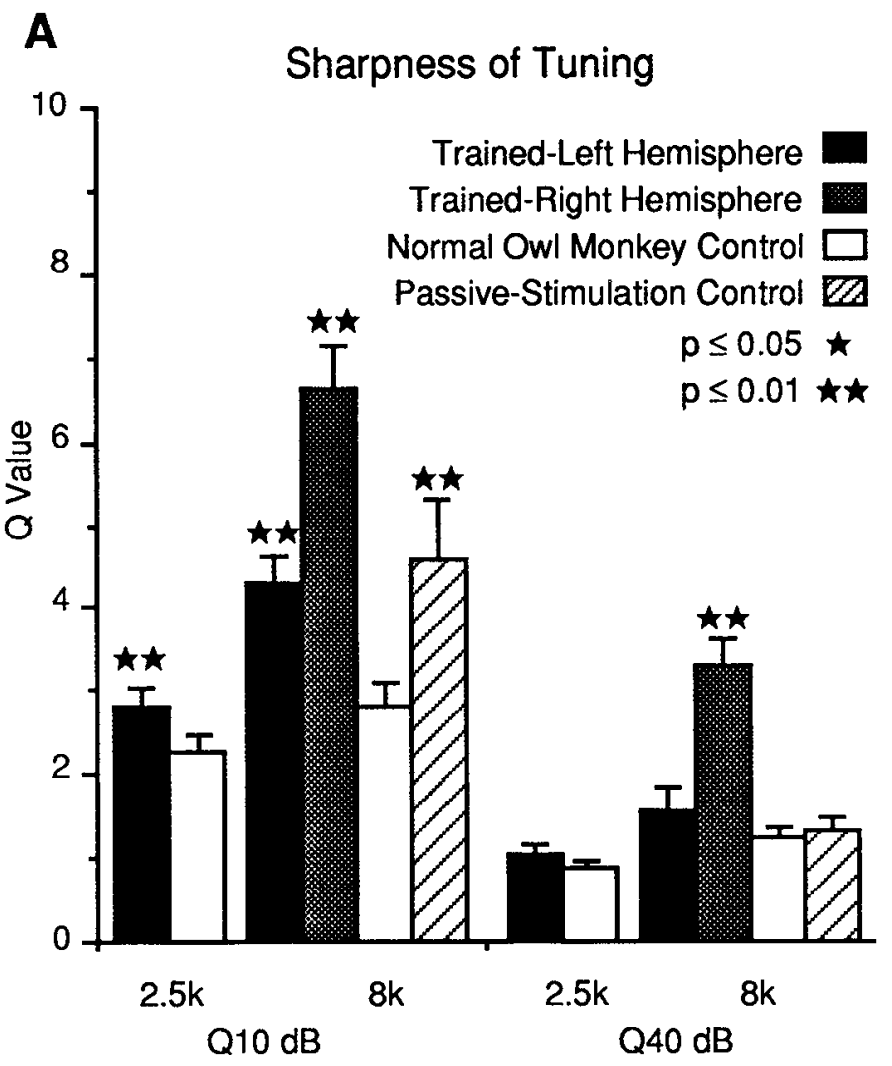

B

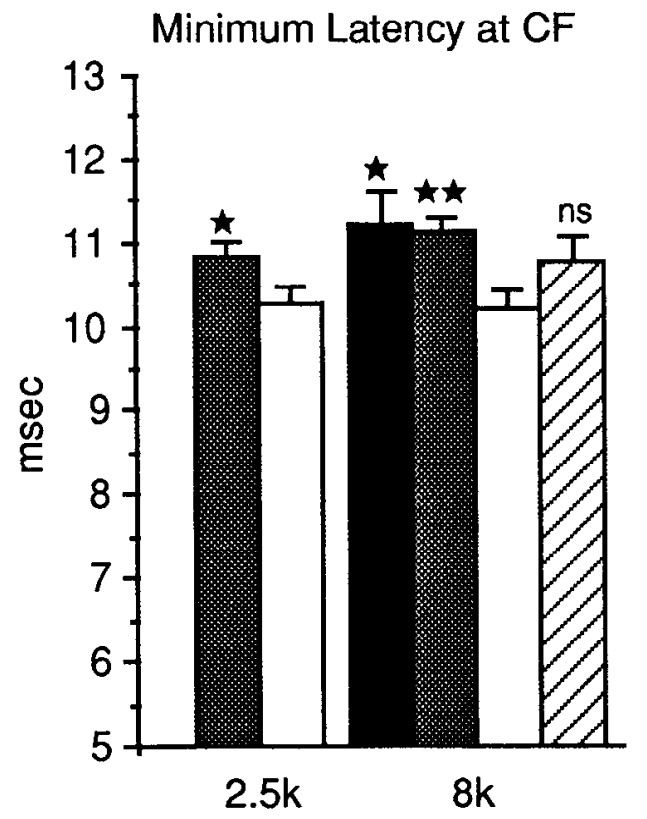

Figure 14. Comparison of sharpness of tuning $(A)$ and minimum latency $(B)$ for different training conditions. Data from each experimental and passive-stimulation hemisphere are shown as a single bar, with the data from the three normal monkeys combined (open bars). Stars indicate statistically significant differences from the normal hemispheres. Data are pooled for all recording locations with CFs between 1.9-3.1 $\mathrm{kHz}(2.5 \mathrm{kHz}$ task) and $6.6-9.9 \mathrm{kHz}(8 \mathrm{kHz}$ task $) . A$, Mean and SE of $\mathrm{Q} 10 \mathrm{~dB}$ (left) and Q40dB (right) for all studied monkeys. B, Minimum latency of the response for the $2.5 \mathrm{kHz}$ (left) and $8 \mathrm{kHz}$ tasks (right).

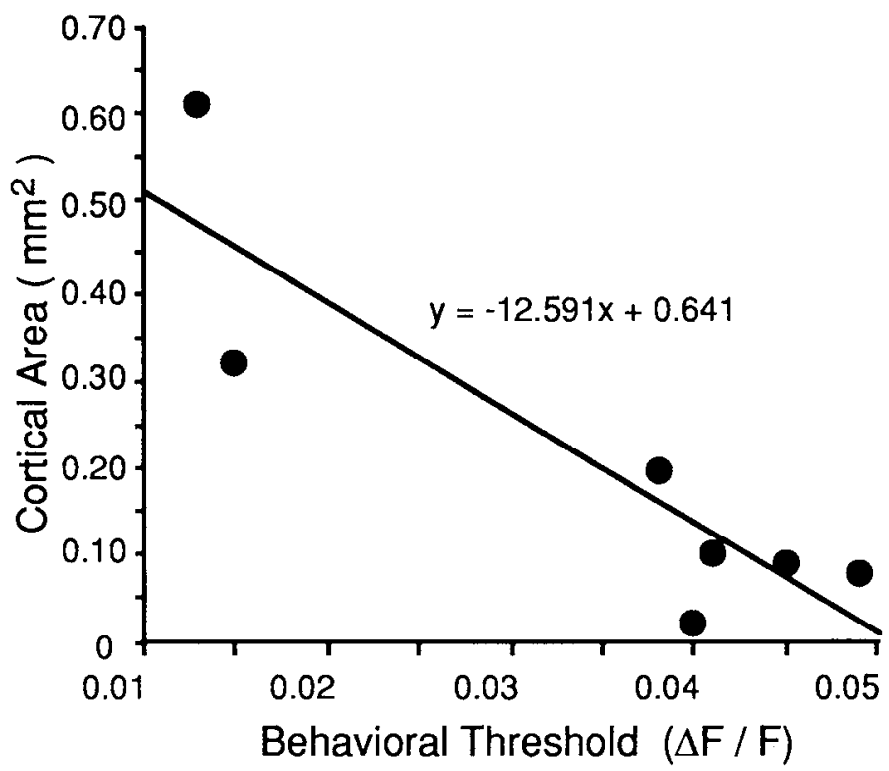

Figure 15. Correlation analysis of the cortical area of representation of the behaviorally trained frequencies at $\mathrm{CF}$ with the behavioral threshold. The equation describes the line with the root mean square best fit to the data $(r=0.882, P<0.01, \mathrm{df}=6)$.

correlated with behavioral frequency discrimination performance.

Behavioral thresholds measured in this study were similar to those described for other nonhuman primates for the $2.5 \mathrm{kHz}$ and $8 \mathrm{kHz}$ tasks (Sinnott et al., 1985, 1987; Prosen et al., 1990), but were higher than those recorded in humans (Wier et al., 1977; Sinnott et al., 1985, 1987). The frequency discrimination thresholds measured for the $3 \mathrm{kHz}$ and $5 \mathrm{kHz}$ tasks were greater than those measured for this frequency range in Old-World monkeys. That may be the result of an attenuation of these middle frequencies in this particular species, for example, by physical constraints imposed by the outer or middle ear. These higher-frequency discrimination thresholds are consistent with the relatively high behaviorally measured detection thresholds in the frequency range of 3-6 kHz in this species (Beecher, 1974), the relatively small cortical representation of this frequency range in AI of normal owl monkeys (Imig et al., 1977; present results), and the higher response thresholds of AI neurons over this frequency range in this and other New-World monkey species (Aitkin et al., 1986; Merzenich et al., 1991).

The electrophysiological experiment revealed a tonotopic organization of owl monkey AI similar to that previously shown in this species (Imig et al., 1977) as well as other primates (Merzenich and Brugge, 1972; Aitken et al., 1986). The responses of neuronal clusters to these tonal stimuli were commonly very brisk, with an initial short-latency burst of activity. In spite of the globally smooth tonotopic organization in AI, the representation of very small frequency ranges was often seen to be patchy for both the experimental and control hemispheres. Part of the explanation is that we were basing the area measurements on the $\mathrm{CF}$ at threshold, and considered only a very small frequency range. It is likcly that this patchy representation would be more continuous if considered from the view of either a higher-intensity stimulus or over a larger frequency range. However, the presence of patches of representation of some frequencies may accurately reflect the tonotopic organization at 
this level of detail, as a similarly discontinuous representation of restricted frequency ranges can be seen in the detailed maps of AI described in the marmoset (Aitkin et al., 1986).

Neural mechanisms of frequency discrimination. The finding that the cortical area of CF representation was correlated with the behavioral performance does not restrict the site of frequency discrimination to that location. Most nuclei throughout the central auditory pathways could contribute to these perceptual distinctions. Studies have indicated that large lesions that include AI do not effect learned frequency discriminations in cats (Butler et al., 1957; Goldberg and Neff, 1961), but deficits have been shown in other behavioral paradigms (Meyer and Woolsey, 1952; Thompson, 1960). These studies suggest that the sequence and timing of stimuli, or perhaps the difficulty of the task, affect the necessary contribution of auditory cortical fields regarding this behavior. The behavioral task used in these experiments cannot be directly compared to those cited above, yet were considered by human observers to be quite difficult and to require vigilant attention in order to perform correctly. An alternative view is that the pairs of tone pips presented sequentially in the present study more closely match auditory pattern discrimination paradigms, which do show deficits following auditory cortical lesions (Diamond and Neff, 1957). A third point to bear in mind is that the limited data available suggest that thalamic areas with frequency-specific responses (ventral medial geniculate nucleus) are less affected by classical conditioning paradigms than the cerebral cortex, although the responses of the more broadly tuned neurons located in the magnocellular division of the medial geniculate are affected by classical conditioning paradigms (see Weinberger et al., 1990, for review). The cortical representations described in this report may he a reflection of tonotopically reorganized subcortical regions, partially reorganized subcortical and cortical areas, or resulting from reorganization only at the cortical level.

Regardless of the fact that we cannot identify the site(s) of reorganization, the differences in the response properties of $\mathrm{AI}$ neurons between trained and untrained monkeys do provide some insights into plausible neural mechanisms of frequency discrimination. Frequency discrimination abilities could potentially be based on either the rate of neuronal discharge, the temporal information encoded within the discharge, and/or the spatial location/distribution of the neurons responding to the different frequencies. Our finding of an increased spatial representation of behaviorally relevant frequencies suggests that the spatial distribution of central neurons at least contributes to this bchavior. One simple hypothesis is that the separation in the spatial locations of appropriately tuned neurons could provide the necessary information to form the basis of the discrimination. Increases in the representation of a frequency range would increase the spatial resolution of those frequencies. The fact that the bandwidth of the tuned response was not significantly different at $40 \mathrm{~dB}$ above threshold suggests that this larger representation was maintained at higher intensities, for example, those used in the behavioral paradigm. However, the cortical area measure at CF described here is almost certainly an oversimplification. By combining the several islands of cortical representation into a single measure, we may have diluted the contributing portion. Studies in the cat have shown that several different parameters of both tuning and timing of the neural response are topographically organized along the isofrequency axis (Mendelson et al., 1988; Schreiner et al., 1988; Schreiner and Mendelson, 1990; Imig et al., 1991; Sutter and Schreiner,
1991), and preliminary data suggest that this is also the case in the owl monkey (Merzenich et al., 1991). It is plausible that only a subset of the total area of representation is contributing to the processing of frequency distinctions, while other parts of the representation are processing other stimulus parameters. Finally, the neural responses were not tested with respect to the their temporal representation of the stimuli. Studies in somatosensory cortex have shown that the occurrences of neural discharges are temporally more coherent in monkeys trained at a tactile frequency discrimination task when compared to unstimulated and passively stimulated monkeys (Recanzone et al., 1992d). The temporal coding of the stimuli could provide additional information regarding frequency.

The finding that Q10dB did increase significantly was unexpected, as the behavioral stimuli were not presented at these low intensities. The disparity between Q10dB and Q40dB suggests that these two parameters are not equivalent, consistent with observations in cat (Schreiner and Mendelson, 1990; Sutter and Schreiner, 1991). Q10dB measures have been reported to covary with latency, and both were observed to increase in the trained monkeys. The longer latencies in the trained monkeys may have resulted from increased neural processing at subcortical levels, or perhaps from a suppression of normally shorterlatency inputs that are involved in processing other aspects of the acoustic signal. These two parameters were not correlated with the behavioral performance, as the higher Q10dB (narrower bandwidths) and latency measures were not matched with the lowest thresholds.

Finally, both hemispheres were investigated in only one monkey. The hemisphere studicd in cach of the other trained monkeys was selected as the hemisphere contralateral to the ear nearest the speaker in the behavioral apparatus. In most cases, the monkey oriented its head either directly toward the speaker, in which case the ears were at an equivalent distance, or toward the food hopper, which would slightly favor one ear over the other. Both hemispheres of OM1 had similar areas of representations; however, there were significant differences between the right and left hemispheres with respect to both $\mathrm{Q} 10 \mathrm{~dB}$ and Q40dB. It is unclear at present how these binaural stimuli are processed by the two hemispheres.

Comparisons with earlier studies. The caveats discussed above do not detract from the findings that there is a significant improvement in behavioral performance at the frequency discrimination task with training that was significantly correlated with the spatial representation of the trained frequencies in primary auditory cortex. Improvements in performance at a perceptual or motor skill have been well documented for a variety of tasks (see Volkmann, 1858; James, 1890; Gibson, 1953; Fitts, 1964), including relatively simple sensory discrimination tasks in adult primates (Sinnott et al., 1985; Prosen et al., 1990; Recanzone et al., 1992a), and have been shown to continue for up to several years of continuous practice. The initial, rapid component of the improvement probably represents a "conceptual" learning of the task and development of the most appropriate strategies (see deJong, 1957; Crossman, 1959; Seibel, 1963; Recanzone et al., 1992a). This is supported by the large variability in the performance between these early sessions, and by the fact that it was not seen when the task was changed to different frequencies in well-trained monkeys (e.g., $2.5 \mathrm{kHz}$ to $8 \mathrm{kHz}$ in OM2).

The second stage of learning was characterized by an improvement in performance at each S2 frequency in a roughly sequential fashion, and an increase in the slope of the psycho- 
metric function at threshold. This improvement has been hypothesized to result from an enhancement of the central representation of the appropriate stimulus parameters (Recanzone et al., 1992a,d). The psychophysical results derived in these monkcys are in agrecment with this hypothesis. For example, there was little transference of the improvement in performance when untrained frequency ranges were tested in widely separated sessions, consistent with studies on absolute auditory detection thresholds (Zwislocki et al., 1958) or tactile frequency discrimination thresholds (Recanzone et al., 1992a). Improvements were only recorded when training was continuous for several days. The representations of these untrained frequencies were comparable to those of normal monkeys. An interesting observation was the apparent decrease in performance for decreasing S2 frequencies $(-\Delta F$ values) with training at increasing $\mathrm{S} 2$ frequencies $(+\Delta F$ values). The thresholds for $-\Delta F$ values have been reported to be slightly lower in humans but vary depending on the species for Old-World monkeys (Sinnott et al., 1987). In those experiments, the behavioral performances were reported following training at both increasing and decreasing $\Delta F$ tasks. In OM4, where several measures of $-\Delta F$ threshold were made, there were no cortical locations observed where the $C F$ was centered in the range of these decreasing $\mathbf{S} 2$ frequencies. Finally, our psychophysical results suggest that there is little, if any, effect of stimulus level on discrimination performance. There was a slight decrease in threshold with increasing stimulus level, but this difference was not statistically significant. Increasing intensity can increase frequency discrimination ability in humans, but decreases performance in macaque monkeys (Sinnott et al., 1987). Our results suggest either that frequency discrimination performance is normally independent of stimulus level in this species, or that the performance at the trained stimulus level improved to match that for lower stimulus levels. The effects of stimulus amplitude on frequency discrimination in a naive animal of this species would provide further information on this issue.

The electrophysiological results are consistent with a variety of preparations in the somatosensory system (for reviews, see Merzenich et al., 1988, 1990; Kaas, 1991) and with the hypothesis that the functional organization of the cortex changes in parallel with perceptual changes (see Merzenich et al., 1988, 1990; Recanzone et al., 1991 b,d; Recanzone and Merzenich, 1992). The finding that the large cortical representation of the behaviorally trained receptor surface was only seen in monkeys trained to discriminate those frequencies, and not in monkeys trained at an unrelated task, is consistent with results from the somatosensory system (Jenkins et al., 1990; Recanzone et al., $1992 \mathrm{~b}-\mathrm{d}$ ). This representational plasticity occurred even though the monkeys in this study were acoustically stimulated for approximately $15-20 \mathrm{~min}$ during the $2 \mathrm{hr}$ session each day, and were allowed to hear other auditory stimuli, some of which were presumably behaviorally significant, for the remaining $22 \mathrm{hr}$ each day. Thus, it is not necessary to present the behaviorally important stimuli to the exclusion of all other stimuli for these effects to occur, and consistent with reports on the effects of classical conditioning on responses of auditory cortical neurons, behavioral relevance of the stimulus is necessary.

The demonstration that there is a larger CF representation in trained monkeys is not altogether unexpected given the frequency-specific changes in neural responses of other auditory cortical areas following classical conditioning paradigms in the cat (Diamond and Weinberger, 1986) and guinea pig (Bakin and
Weinberger, 1990) and changes in the tonotopic representation of AI after restricted cortical lesions in the adult guinea pig and cat (Robertson and Irvine, 1989; Irvine et al., 1991). In contrast to the conditioning studies, there was no obvious change in the overall responsiveness of neurons representing the frequencies used in behavioral training, and there was no significant change in the tuning of these neurons at intensities well above threshold. Similarly, several months after restricted lesions in the cochlea, the overall activity of neurons in the expanded zone of $\mathrm{CF}$ representation in $\mathrm{AI}$ is similar to neurons in the adjacent, unaffected region. These differences may reflect differences in the comparison between short-term effects as in the conditioning studies and the long-term effects as in this and the lesion studies, in the anesthetic state of the animal, or in the differences between the conditioning and discrimination behavioral paradigms.

Several models advanced to account for cortical plasticity have proposed that synaptic efficacies are altered consequent to the temporal correlation of pre- and postsynaptic activity by a Hebbian mechanism (e.g., see Edelman, 1987; Merzenich et al., 1988, 1990; von der Malsburg and Singer, 1988; Palm, 1990; Singer, 1990; Weinberger ct al., 1990). These mechanisms, although presented with respect to the cerebral cortex, could be equally effective for representational changes in subcortical areas. Computer models using these types of synaptic efficacy changes result in changes in topography similar to those observed in the somatosensory (Pearson et al., 1987; Grajski and Merzenich, 1990) and visual cortices (Miller et al., 1989). The present results are consistent with models of this class, as the increased area of representation and the increased sharpness of tuning could result from the strengthening of the synapses representing the behaviorally trained frequencies, and a weakening of synapses representing other, neighboring frequencies. The unaffected representations in the passive stimulation monkeys suggest that attention to the stimulus enhances the response, perhaps via neuromodulators such as $\mathrm{ACh}$ or norepinephrine (see Singer, 1990; Weinberger et al., 1990).

The inverse relationship between receptive field size and the cortical area of representation described in the visual and somatosensory systems (Hubel and Wiesel, 1974; Sur et al., 1980) is thought to arise by competitive interactions of excitatory and inhibitory inputs between cortical neurons comprising a horizontally oriented network (see Edelman, 1987; Merzenich et al., 1990). These local interactions between cortical neurons would also restrict the improvement to the behaviorally trained and immediately surrounding frequencies, and thus only limited effects on either the cortical representation or behavioral performance for other frequencies are expected. The decreasing performance at $-\Delta F$ frequencies observed in this study suggests that these horizontal influences degrade the representation of unused neighboring frequencies, perhaps by "borrowing" territory from the representation of those frequencies. The local interactions would also be expected to sharpen the temporal cohesiveness of the responses over a wider area, as has been demonstrated in the somatosensory cortex (Recanzone et al., 1992d), which would result in a stronger representation of the stimulus in both space and time that could then be transmitted to other areas for further processing.

Technical considerations. Several technical considerations must be remembered when attempting to correlate data derived by presenting single tone bursts in a closed sound system in an anesthetized monkey with behavioral measures performed using sequential tone bursts in the free field. The barbiturate anes- 
thesia may have masked differential neuronal responses to behaviorally relevant stimuli. The effects of barbiturate anesthesia on tuning properties of cells in AI of the monkey are not well described; however, comparison of our data with that of others derived in awake animals (i.e., Funkenstein and Winter, 1973; Pelleg-Toiba and Wollberg, 1989) suggests little effect on measures of CF or temporal response properties of cortical neurons. In this study, all of the comparisons of the neural responses were made between monkeys anesthetized in a similar manner; thus, all anesthetic effects are constant and do not differentially affect one set of monkeys.

A second consideration is that the stimuli used in generating the cortical maps were shorter in duration $(50 \mathrm{msec}$ vs. 150 $\mathrm{msec}$ ) and were presented through a closed sound system. The acoustic environment in the free field and the resonance properties of the external ear may have distorted the stimuli in the behavioral condition. The definition of response properties of AI neurons using a closed sound system has the advantage of better control of the stimulus intensity within and between individual experiments. Direct comparisons can also be made between these results and those of others. If the electrophysiological experiments were performed in the free field, idiosyncratic distortions of the stimulus could occur between individual monkeys due to variations in head size and location and pinna morphology. To match the acoustic environment of the behavioral apparatus in the electrophysiology experiment, it would have been necessary to perform both in the same apparatus, which was not possible. Finally, the technique we have used is appropriate to achieve the primary goal of the study, namely, to define alterations in the tonotopic organization of $\Lambda \mathrm{I}$ consequent to the behavioral training.

A third potential problem is in correlating the pooled data from normal monkeys with the behavioral results from other monkeys either early in training or when tested on untrained frequencies. This method increased the number of data points such that reasonable correlation analysis between physiological and behavioral data could be performed, but is based on the assumption that the physiological and behavioral measures are representative for all monkeys. The estimates of "untrained" behavioral performance are probably reasonable because the thresholds for sessions 5-10 in monkey OM1 were within 15\% of the initial five sessions for OM2 at the $8 \mathrm{kHz}$ task, as were the measures for sessions 5-10 for monkeys OM2 and OM3 in the $2.5 \mathrm{kHz}$ task. These differences were not significantly different, and all of our data for both auditory and tactile frequency discrimination measures using this paradigm show low interindividual variability (see Recanzone et al., 1991, 1992a). There was also some variability in the cortical representations between normal monkeys, as has been seen previously in AI (Merzenich and Brugge, 1973; Imig et al., 1977; Aitken et al., 1986; see also Merzenich et al., 1975), consistent with interanimal variability described in primary somatosensory cortex (Merzenich et al., 1987). This variability was most evident in the large representation of frequencies in the range of $10 \mathrm{kHz}$ in monkey $\mathrm{N} 2$ and for frequencies near $11 \mathrm{kHz}$. in monkey CM 1 . This interanimal variability of frequency representations was reduced when considered from the point of view of the expanded representations in the trained monkeys, where cortical representations were invariably several times greater than the mean representations from untrained hemispheres. These idiosyncrasies of primate AI organization prompted us to utilize the strategy of using different comparison frequencies for each of the limited number of owl monkeys trained in the behavioral task. Obviously, if one had trained monkey $\mathrm{N} 2$ on a $10 \mathrm{kHz}$ task, they may have been misled by the preexisting large representation of those frequencies. By the same logic, the probability of matching the frequency in the behavioral paradigm with the idiosyncratically overrepresented frequency in each of the studied individual monkeys is remote.

One potential inadequacy of the data is that frequency response areas, and hence the measures of Q10dB and Q40dB, were based on the single presentation of each of the 675 stimuli. Spontaneous activity occurring in the $50 \mathrm{msec}$ time window of data collection could artificially broaden the response area, and only stimuli that evoke a response on several repeated trials might be consistently considered within the response area. However, this method has been shown to be a reliable measure of multiple-unit responses. The objective criteria used to define Q10dB and Q40dB take into account the rate of spontaneous activity as well as the consistency of the response latency, and have been argued to accurately reflect the tuning and timing properties of the neurons under study (see Schreiner and Mendelson, 1990; Sutter and Schreiner, 1991). The minimum latency estimate was based on all 15 levels at three frequencies, and therefore represents 45 separate measurements.

Concluding remarks. The demonstration that the extent of the cortical area of representation of a specific frequency range is correlated with the behaviorally measured discrimination performance represents a first approximation for determining the neural correlate of this perceptual judgement. The results of these experiments are consistent with hypotheses that auditory cortical representations are modifiable and that this plasticity reflects the acquisition of skills and behaviors throughout life. A key prediction from these hypotheses is that the changes in cortical representation should occur in parallel with the behavioral gains, and repeated measures of these representations should correlate with the repeated measures of behavioral performance. A second prediction is that inactivation of restricted areas of the central representation of these stimuli should result in restricted behavioral deficits. Finally, the effects of interrupting the normal release of putative neuromodulators during the period of acquisition of this behavioral improvement could begin to reveal the pharmacology of this representational plasticity as has been done in other systems (i.e, Kasamatsu and Pettigrew, 1979; Bear and Singer, 1986). Such studies would provide key evidence in extending these hypotheses and models of cortical function.

\section{References}

Aitkin LM, Merzenich MM, Irvine DRF, Clarey JC, Nelson JE (1986) Frequency representation in auditory cortex of the common marmoset (Callithrix jacchus jacchus). J Comp Neurol 252:175-185.

Ashe JH, McKenna TS, Weinberger NM (1989) Cholinergic modulation of frequency receptive fields in auditory cortex. II. Frequencyspecific effects of anticholinesterases provide evidence for a modulatory action of endogenous ACh. Synapse 4:44-54.

Bakin JS, Weinberger NM (1990) Classical conditioning induces CSspecific receptive field plasticity in the auditory cortex of the guinea pig. Brain Res 536:271-286.

Bear MF, Singer W (1986) Modulation of visual cortical plasticity by acetylcholine and noradrenaline. Nature 320:172-176.

Beecher MD (1974) Hearing in the owl monkey (Aotus trivirgatus). I. Auditory sensitivity. J Comp Physiol Psychol 86:898-901.

Butler RA, Diamond IT, Neff WD (1957) Role of auditory cortex in discrimination of changes in frequency. J Neurophysiol 20:108-120.

Craig JC (1988) The role of experience in tactual pattern perception: a preliminary report. Int J Rehabil Res 11:167-171. 
Crossman ERFW (1959) A theory of the acquisition of speed skill. Ergonomics 2:153-166.

deJong JR (1957) The effects of increasing skill on cycle time and its consequences for time standards. Ergonomics 1:51-60.

Diamond DM, Weinberger NM (1986) Classical conditioning rapidly induces specific changes in frequency receptive fields of single neurons in secondary and ventral ectosylvian auditory cortical fields. Brain Res 372:357-360.

Diamond IT, Neff WD (1957) Ablation of temporal cortex and discrimination of auditory patterns. J Neurophysiol 20:300-315.

Disterhoft JF, Stuart DK (1976) Trial sequence of changed unit activity in auditory system of alert rat during conditioned response acquisition and extinction. J Neurophysiol 39:266-281.

Edelman GM (1987) Neuronal Darwinism: the theory of neuronal group selection. New York: Basic.

Fitts PM (1964) Perceptual-motor skill learning. In: Categories of human learning (Melton AW, ed), pp 243-285. New York: Academic.

Funkenstein HH, Winter P (1973) Responses to acoustic stimuli of units in the auditory cortex of awake squirrel monkeys. Exp Brain Res 18:464-488.

Gibson EJ (1953) Improvement in perceptual judgments as a function of controlled practice or training. Psychol Bull 50:401-431.

Goldberg JM, Neff WD (1961) Frequency discrimination after bilateral ablation of cortical auditory areas. J Neurophysiol 24:119-128.

Grajski KA, Merzenich MM (1990) Hebb-type dynamics is sufficient to account for the inverse magnification rule in cortical somatotopy. Neural Comput 2:71-84.

Green DM, Swets IA (1966) Signal detection theory. New York: Wiley.

Hubel DH, Wiesel TN (1974) Uniformity of monkey striate cortex: a parallel relationship between field size, scatter and magnification factor. J Comp Neurol 158:295-302.

Imig TJ, Rugero MA, Kitzes LM, Javel E, Brugge JF (1977) Organization of auditory cortex in the owl monkey (Aotus trivirgatus). $\mathrm{J}$ Comp Neurol 171:111-128.

Imig TJ, Irons A, Samson FR (1991) Single-unit selectivity to azimuthal direction and sound pressure level of noise bursts in cat highfrequency primary auditory cortex. J Neurophysiol 63:1448-1466.

Irvine DRF, Rajan R, Wize LZ, Heil P (1991) Reorganization in auditory cortex of adult cats with unilateral restricted cochlear lesions. Soc Neurosci Abstr 17:1485.

James W (1890) The principles of psychology, Vol I. New York: Dover.

Jenkins WM, Merzenich MM, Ochs MT, Allard T, Guíc-Robles E (1990) Functional reorganization of primary somatosensory cortex in adult owl monkeys after behaviorally controlled tactile stimulation. J Neurophysiol 63:82-104.

Kaas JH (1991) Plasticity of sensory and motor maps in adult mammals. Annu Rev Neurosci 14:137-167.

Kasamatsu T, Pettigrew JD (1979) Preservation of binocularity after monocular deprivation in the striate cortex of kittens treated with 6-hydroxydopamine. J Comp Neurol 185:139-162.

Kitzes LM, Farley GR, Starr A (1978) Modulation of auditory cortex unit activity during the performance of a conditioned response. Exp Neurol 62:678-697.

McKenna TM, Ashe JH, Weinberger NM (1989) Cholinergic modulation of frequency receptive fields in auditory cortex. I. Frequencyspecific effects of muscarinic agonists. Synapse 4:30-43.

Mendelson JR, Schreiner CE, Grasse K, Sutter ML (1988) Spatial distribution of responses to FM sweeps in cat primary auditory cortex. Abstr Assoc Res Otolaryngol 11:36.

Merzenich MM, Brugge JF (1973) Representation of the cochlear partition on the superior temporal plane of the macaque monkey. Brain Res 50:275-296.

Merzenich MM, Knight PL, Roth GL (1975) Representation of cochlea within primary auditory cortex in the cat. J Neurophysiol 38:231249.

Merzenich MM, Nelson RJ, Kaas JH, Stryker MP, Jenkins WM, Zook JM, Cynader MS, Schoppmann A (1987) Variability in hand surface representations in areas $3 b$ and 1 in adult owl and squirrel monkeys. J Comp Neurol 258:281-396.

Merzenich MM, Recanzone G, Jenkins WM, Allard TT, Nudo RJ (1988) Cortical representational plasticity. In: Neurobiology of neocortex (Rakic P, Singer W, eds), pp 41-67. New York: Wiley.

Merzenich MM, Recanzone GH, Jenkins WM, Nudo RJ (1990) How the brain functionally rewires itself. In: Natural and artificial parallel computations (Arbib M, Robinson JA, eds), pp 177-210. Cambridge, MA: MIT Press.

Merzenich MM, Schreiner CE, Recanzone GH, Beitel RE, Sutter ML (1991) Topographic organization of cortical field AI in the owl monkey Aotus trivirgatus. Abstr Assoc Res Otolaryngol 14:44.

Metherate R, Weinberger NM (1990) Cholinergic modulation of responses to single tones produces tone-specific receptive field alterations in cat auditory cortex. Synapse 6:133-145.

Meyer DR, Woolsey CN (1952) Effects of localized cortical destruction on auditory discriminative conditioning in cat. J Neurophysiol 15: 149-162.

Miller KD, Keller JB, Stryker MP (1989) Ocular dominance column development: analysis and simulation. Science 245:605-615.

Olds J, Disterhoft JF, Segal M, Kornblith CL, Hirsh R (1972) Learning centers of rat brain mapped by measuring latencies of conditioned unit responses. J Neurophysiol 35:202-219.

Palm G (1990) Cell assemblies as a guideline for brain research. Concepts Neurosci 1:133-147.

Pearson JC, Finkel LH, Edelman GM (1987) Plasticity in the organization of adult cerebral cortical maps: a computer simulation based on neuronal group selection. J Neurosci 7:4209-4223.

Pelleg-Toiba R, Wollberg Z (1989) Tuning properties of auditory cortex cells in the awake squirrel monkey. Exp Brain Res 74:353-364.

Prosen CA, Moody DB, Sommers MS, Stebbins WC (1990) Frequency discrimination in the monkey. J Acoust Soc Am 88:2152-2158.

Recanzone GH, Merzenich MM (1992) Alterations of the functional organization of primary somatosensory cortex following intracortical microstimulation or behavioral training. In: Memory: organization and locus of change (Squire LM, Weinberger NM, Lynch G, McGaugh JL, eds), pp 217-238. New York: Oxford UP.

Recanzone GH, Jenkins WM, Hradek GT, Merzenich MM (1991) A behavioral frequency discrimination paradigm for use in adult primates. Behav Res Methods Instrum Comput 23:357-369.

Recanzone GH, Jenkins WM, Hradek GT, Merzenich MM (1992a) Progressive improvements in discriminative abilities in adult owl monkeys performing a tactile frequency discrimination task. J Neurophysiol 67:1015-1030.

Recanzone GH, Merzenich MM, Jenkins WM, Grajski KA, Dinse HR (1992b) Topographic reorganization of the hand representation in cortical area $3 \mathrm{~b}$ of owl monkeys trained in a frequency discrimination task. J Neurophysiol 67:1031-1056.

Recanzone GH, Merzenich MM, Jenkins WM (1992c) Frequency discrimination training engaging a restricted skin surface results in an emergence of a cutaneous response zone in cortical area 3a. J Neurophysiol 67:1057-1070.

Recanzone GH, Merzenich MM, Schreiner CE (1992d) Changes in the distributed temporal response properties of SI cortical neurons reflect improvements in performance on a temporally-based tactile discrimination task. J Neurophysiol 67:1071-1091.

Robertson D, Irvine DRF (1989) Plasticity of frequency organization in auditory cortex of guinea pigs with partial unilateral deafness. $\mathbf{J}$ Comp Neurol 282:456-471.

Ryugo DK, Weinberger NM (1978) Differential plasticity of morphologically distinct populations in the medial geniculate body of the cat during classical conditioning. Behav Biol 22:275-301.

Schreiner CE, Mendelson JR (1990) Functional topography of cat primary auditory cortex. Distribution of integrated excitation. J Neurophysiol $64: 1442-1459$.

Schreiner CE, Mendelson JR, Grasse K, Sutter ML (1988) Spatial distribution of basic response properties in cat primary auditory cortex. Abstr Assoc Res Otolaryngol 11:36.

Seibel R (1963) Discrimination reaction time for a 1,023-alternative task. J Exp Psychol 66:215-226.

Singer W (1990) Search for coherence: a basic principle of cortical self-organization. Concepts Neurosci 1:1-26.

Sinnott JM, Petersen MR, Hopp SL (1985) Frequency and intensity discrimination in humans and monkeys. J Acoust Soc Am 78:19771985.

Sinnott JM, Owren MJ, Petersen MR (1987) Auditory frequency discrimination in primates: species differences (Cercopithecus, Macaca, Homo). J Comp Psychol 101:126-131.

Sur M, Merzenich MM, Kaas JH (1980) Magnification, receptive-field area, and "hypercolumn" size in areas $3 b$ and 1 of somatosensory cortex in owl monkeys. J Neurophysiol 44:295-311.

Sutter ML, Schreiner CE (1991) Physiology and topography of neurons 
with multipeaked tuning curves in cat primary auditory cortex. J Neurophysiol 65:1207-1226.

Thompson RF (1960) Function of auditory cortex of cat in frequency discrimination. J Neurophysiol 23:321-334.

Volkman AW (1858) Über den Einfluss der Übung. Leipzig Ber MathPhys Classe 10:38-69.

von der Malsburg C, Singer W (1988) Principles of cortical network organization. In: Neurobiology of neocortex (Rakic P, Singer W, eds), pp 69-99. New York: Wiley.

Weinberger NM, Ashe JH, Metherate R, McKenna TM, Diamond DM, Bakin J (1990) Returning auditory cortex by learning: a preliminary model of receptive field plasticity. Concepts Neurosci 1:91-132.
Wier CC, Jesteadt W, Green DM (1977) Frequency discrimination as a function of frequency and sensation level. J Acoust Soc Am 61: 178-184.

Wong-Riley MTT, Merzenich MM, Leake PS (1978) Changes in endogenous enzymatic reactivity to DAB induced by neuronal inactivity. Brain Res 141:185-192.

Zwislocki J, Maire F, Feldman AS, Rubin H (1958) On the effect of practice and motivation on the threshold of audibility. J Acoust Soc Am 30:254-262. 